\title{
ESSAI D'ANALYSES QUANTITATIVE, QUALITATIVE ET BIOGÉOGRAPHIQUE DE LA FLORE DU BOU- NACEUR (MOYEN-ATLAS ORIENTAL, MAROC)
}

\author{
Abdelilah RAHOU
}

RÉSUMÉ. Essai d'analyses quantitative, qualitative et biogéographique de la flore du BouNaceur (Moyen-Atlas oriental, Maroc). En l'absence d'étude exhaustive sur le Moyen-Atlas oriental, la flore de cette région est restée assez mal connue par rapport au reste du Maroc. Des recherches sur la flore et la végétation ont été intéressantes sur le massif du Bou-Iblane proche du massif du Bou-Naceur. Mais ce dernier est resté jusqu'à présent moins étudié sur le plan botanique. L'inventaire floristique du Bou-Naceur actuellement évalué à 557 espèces et sousespèces paraît bien important eu égard à la surface trop restreinte du massif. Dans cet article, nous rappelons brièvement le bioclimat du Bou-Naceur et nous mettons en lumière les récentes connaissances de sa flore et de sa biogéographie.

Mots clés. Maroc, Moyen-Atlas oriental, Bou-Naceur, flore, biogéographie.

RESUMEN. Análisis cuantitativo, cualitativo y biogeográfico de la flora de Bou-Naceur. (Atlas Medio Oriental, Marruecos). En ausencia de un estudio exaustivo sobre el Atlas Medio Oriental, la flora de esta región es poco conocida en relación al resto de Marruecos. Investigaciones sobre la flora y vegetación del Macizo de Bou-Iblane próximo al Macizo de Bou-Naceur, han sido interesantes, sin embargo éste último ha sido poco estudiado desde el punto de vista botánico. El inventario florístico de Bou-Naceur actualmente comprende 557 taxones, un elevado número en relación a su escasa superficie. En este artículo, se aportan datos sobre el bioclima, biogeografía y flora de Bou-Naceur.

Palabras clave. Marruecos, Atlas Medio Oriental, Bou-Naceur, flora, biogeografía.

\section{INTRODUCTION}

Afin de mieux connaître le massif du BouNaceur, une liste floristique relative à ses espèces vasculaires est donnée pour la première fois dans ce travail. Ces espèces ont été notées d'après 245 relevés répartis un peu partout dans le massif. Notre inventaire comprend beaucoup de taxons nouveaux pour la région du moins si l'on se réfère au Catalogue des plantes du Maroc "CPM" (Jahandiez \& Maire, 1931, 1932, 1934 et Emberger \& Maire, 1941). D'un autre côté, nous y avons inclus des espèces qui étaient signalées auparavant notamment par Quezel (1957), mais que nous n'avons pas rencontré sur le terrain. Le Catalogue ainsi établi peut être considéré comme assez exhaustif. Outre son intérêt sur le plan inventaire, c'est également 
un outil indispensable qui permet d'aborder les aspects chorologique et biogéographique pour mieux apprécier l'intérêt floristique du BouNaceur à l'échelle du Maroc.

\section{MATÉRIEL ET MÉTHODE}

La région étudiée est située dans l'extrémité orientale du Moyen-Atlas et occupe une surface d'environ $1600 \mathrm{~km}^{2}$. Elle est limitée au nord-est par la plaine de Guércif, au nord par le massif de Bou-Iblane, au sud par le bassin de Moulouya et à l'ouest par le synclinal perché de l'oued Chegg el-Ard. Elle correspond aux bassins des oueds Bou-Rached, Zobzit, Beni-Mansour, Soufoulout et aux chaines montagneuses de Ouizzoukane (2350 mètres), de Bou-Naceur (3340 m) et de Guelb er-Rahal (3157 m).

Située entre les latitudes $33^{\circ} 20^{\prime} \mathrm{N}$ et $33^{\circ} 40^{\prime} \mathrm{N}$, la région est soumise à un climat typiquement méditerranéen caractérisé par un été chaud et sec et un hiver froid et pluvieux. Il existe très peu de données chiffrées de pluie et surtout de température. L'analyse thermique repose sur des estimations référées à des gradients altitudinaux à partir de certains postes climatiques voisins. Les précipitations sont dues la plupart du temps à des courants d'air humides venant de l'ouest et du sud-ouest. Aux altitudes supérieures à 2000 mètres, les précipitations neigeuses sont importantes. La comparaison des données de différents postes a permis de déterminer les gradients pluviométriques suivants: un gradient croissant sur les revers nord-ouest du massif d'environ 58 millimètres $(\mathrm{mm})$ de pluie par 100 mètres (m) d'altitude; un gradient croissant sur les revers sud d'environ $15 \mathrm{~mm} / 100 \mathrm{~m}$; un gradient croissant de l'est vers l'ouest de $65 \mathrm{~mm} / 100$ m.

Les zones les plus humides sont localisées dans la partie occidentale du massif par contre la moitié orientale est assez sèche. La quantité de pluie peut-être inférieure à 200 millimètres par an sur les revers sud.

Il est bien évident que le climat n'est pas uniforme sur tout le territoire, il est fortement influencé par un certain nombre de facteurs locaux tels que l'orographie et l'exposition. Nous pouvons dire que la dition rassemble une gamme d'ambiances bioclimatiques se composant de l'aride, du semi-aride, du subhumide et du bioclimat des hauts sommets (sensu Emberger, 1939). L'humide et l'aride sont faiblement représentés.

En nous basant sur nos observations personnelles, nous avons déterminé six types biologiques de la flore du massif: phanérophytes $(\mathrm{Ph})$, nanophanérophytes (Nph), chaméphytes (Ch), hémicryptophytes (Hém), thérophytes (Th) et géophytes $(\mathrm{G})$.

Les relevés floristiques ont été effectués suivant des transects répartis sur l'ensemble du massif. Ces transects ont été choisis en fonction des principaux gradients climatiques repérés dans la région et des répartitions des formations végétales. Un relevé a été réalisé chaque fois que le milieu change par sa physionomie, sa composition floristique ou ses paramètres écologiques. Le travail de détermination des noms scientifiques, réalisé en grande partie à l'aide de la Nouvelle Flore de l'Algérie "NFA" (Quezel \& Santa 1962. 63), a été vérifié à l'herbier national abrité à l'Institut Scientifique de Rabat. La nomenclature adoptée est celle de Med-Cheklisî "Mchl" (Greuter et al., 1984, 1986, 1989) ou à défaut celle du CPM.

Pour les aspects chorologique et biogéographique, le massif a été subdivisé en différentes unités jugées homogènes sur le plan floristique et écologique. Ainsi en superposant la carte de végétation du Bou-Naceur à une carte topographique à la même échelle, nous avons repéré des unités floristico-écologiques. Ensuite, chacune de ces unités majeures a été découpée en plusieurs sous-unités selon les gradients climatiques et la répartition des 
principales formations végétales de la dition.

Pour chaque espèce, nous donnons le type biologique, l'étage bioclimatique et sa répartition dans le massif et au Maroc.

\section{RÉSULTATS}

Au total, 245 relevés ont été exécutés groupant 557 espèces et sous-espèces classées en 283 genres et 68 familles (voir Annexe).

Le nombre d'espèces et sous espèces du Bou-Naceur représente environ 9\% de la flore vasculaire du Maroc inventoriée jusqu'à présent (cf. Fennane \& Ibn-Tatou 1999).

Parmi les familles les plus riches en espèces, nous citons par ordre d'importance les Asteraceae, les Fabaceae, les Brassicaceae, les Poaceac, les Lamiaceae, les Caryophyllaceae, les Scrophulariaceae, les Apiaceae, les Rubiaceae et les Rosaceae. Elles contiennent respectivement $102,52,51,43$, $38,36,18,17,15$ et 12 espèces du massif. Les deux dernières familles sont mieux représentées dans le Bou-Naceur qu'à l'échelle du pays.

Au niveau des genres, Astragalus et Galium occupent la première place avec 10 espèces. Nous comptons aussi 9 espèces du genre Silene et 8 espèces pour chacun des genres Teucrium et Centaurea. Le rang de 7 espèces est occupé par Euphorbia, Linaria et Plantago. Celui de 6 et 5 espèces est donné respectivement à Alyssum, Avena, Ononis et Stipa. En général, le classement des genres est loin d'être le même entre la flore du massif et celle globale du Maroc.

D'autre part, la liste de la flore du BouNaceur est assez riche en taxons endémiques du Maroc, elle comprend au total 60 espèces, 19 sous-espèces et 13 variétés, ce qui représente $17 \%$ de la flore totale du Bou-Naceur et $11 \%$ des endémiques du pays.

\section{Spectre biologique}

Le spectre biologique établi pour la flore de Bou-Naceur est à prédominance hémicryptophytique $(33 \%)$ et thérophytique (30\%). Les chaméphytes représentent $22 \%$ alors que les géophytes sont de moindre importance $(6 \%)$. Les faibles proportions des phanérophytes $(3 \%)$ et des nanophanérophytes $(6 \%)$ traduisent une certaine homogénéité des strates arborées et arbustives des forêts (tab. 1).

L'analyse du spectre biologique établi pour certaines formations végétales montre une prédominance thérophytique puis chaméphytique dans la steppe, la tétraclinaie et la junipéraie rouge, et hémicryptophytique dans la chênaie verte, la cédraie et la xérophytaie.

Ces variations sont également en corrélation avec le bioclimat. En effet le spectre biologique établi par étages bioclimatiques déterminés dans le massif montre que les taux des thérophytes et des hémicryptophytes

\begin{tabular}{lcccccc}
\hline & $\begin{array}{c}\text { Steppe à alfa } \\
\text { Stipa } \\
\text { tenacissima) }\end{array}$ & $\begin{array}{c}\text { Tétraclinaie } \\
\text { (Tetraclinis } \\
\text { articulata) }\end{array}$ & $\begin{array}{c}\text { Junipéraie } \\
\text { rouge } \\
\text { (Juniperus } \\
\text { phoenicea) }\end{array}$ & $\begin{array}{c}\text { Chênaie } \\
\text { verte } \\
\text { Quercus } \\
\text { ilex) }\end{array}$ & $\begin{array}{c}\text { Cédraie } \\
\text { (Cedrus } \\
\text { atlantica) }\end{array}$ & $\begin{array}{c}\text { Xérophytaie } \\
\text { (formation à } \\
\text { xérophytes épineux) }\end{array}$ \\
\hline Thérophyte & $\mathrm{U} 4$ & $\mathrm{U} 3$ & $\mathrm{U} 3$ & $\mathrm{U} 3$ & $\mathrm{U} 2$ & $\mathrm{U1}$ \\
Hémicryptophyte & $40.8 \%$ & $43.9 \%$ & $36.8 \%$ & $21.2 \%$ & $19.2 \%$ & $09.1 \%$ \\
Géophyte & $18.8 \%$ & $13.3 \%$ & $15.8 \%$ & $34.3 \%$ & $37.4 \%$ & $48.5 \%$ \\
Chaméphyte & $4.7 \%$ & $00.0 \%$ & $04.2 \%$ & $04.0 \%$ & $04.0 \%$ & $02.0 \%$ \\
Nanophanérophyte & $23.5 \%$ & $24.5 \%$ & $28.4 \%$ & $28.3 \%$ & $29.3 \%$ & $32.3 \%$ \\
Phanérophyte & $11.0 \%$ & $09.2 \%$ & $11.6 \%$ & $08.1 \%$ & $07.1 \%$ & $06.1 \%$ \\
& $1.2 \%$ & $09.1 \%$ & $03.2 \%$ & $04.1 \%$ & $3.1 \%$ & $02.0 \%$ \\
\hline
\end{tabular}

Tableau 1. Spectre biologique des principales formations végétales de Bou-Naceur. Pour les abreviations des unités, voir texte. 


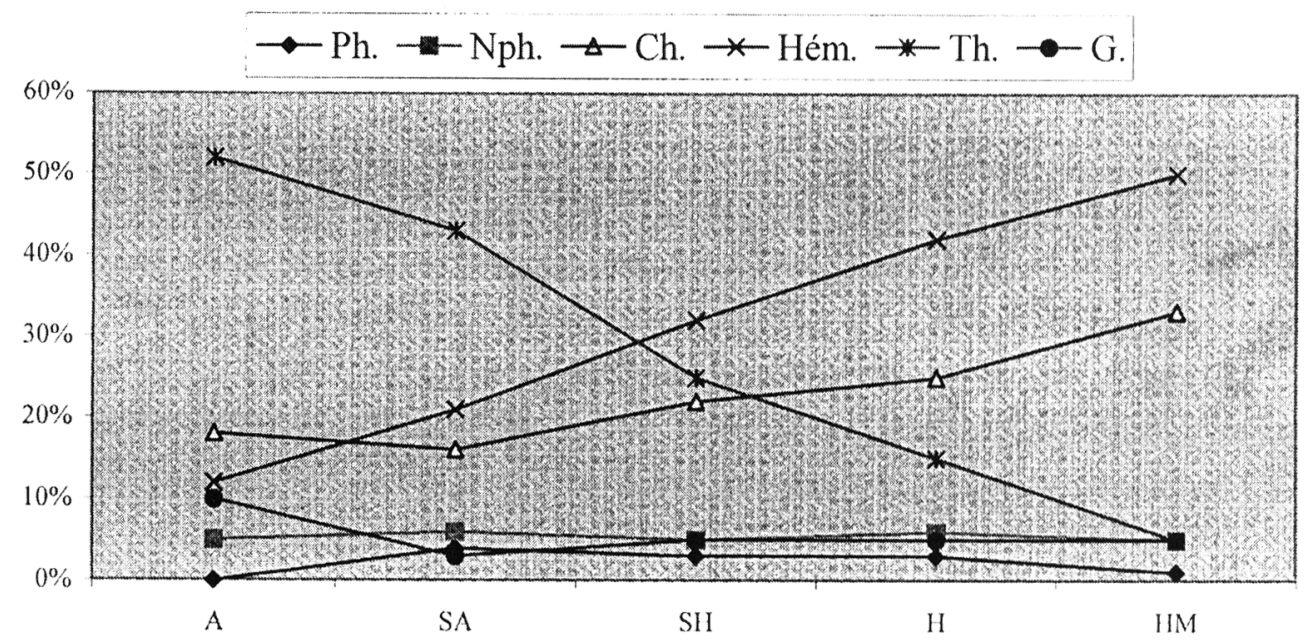

Figure 1. Spectre biologique de la flore de Bou-Naceur par étage bioclimatique; A: aride; SA: semi-aride; SH: sub-humide; H: humide; HM: haute montagne.

évoluent en sens inverse. Le taux des thérophytes baisse lorsqu' on monte en altitude alors que celui des hémicryptophytes augmente (fig. 1). Ces deux types s'épanouissent dans des conditions écologiques absolument différentes.

\section{Unités floristico-écologiques du Bou- Naceur}

En fonction des données physiques (substrat, climat, altitud) et biotiques (nature des formations végétales dominantes) et grâce à notre grande connaissance du terrain nous avons pu distinguer dans la région quatre grandes unités floristico-écologiques que nous présentons brièvement ci-dessous (fig. 2).

Unité 1 (U1): Unité des sommets froids caractérisée essentiellement par des xérophytes épineux, elle est subdivisée en fonction du gradient climatique latéral à humidité décroissante d'ouest à l'est en sous unités U1A, U1B et U1C.

Unité 2 (U2): Elle regroupe les zones les plus arrosées du massif caractérisées par des chênaies et des cédraies ; Elle est découpée en fonction des gradients climatiques et de l'exposition en sous unités:-U2A est caractérisée par des chênaies et des cédraies
bienvenantes;-U2B regroupe les monts qui séparent les hauts sommets du Bou-Naceur et du Bou-Iblane. Elle est dominée par des chênaies et des cédraies assez ouvertes;-U2C concerne le bassin de l'oued Beni-Mansour où s'installe un mélange de chêne vert et de genévrier rouge;-U2D est couverte par une chênaie claire mélangée au genévrier rouge et -U2E, en exposition sud, présente des chênaies très clairsemées et assez pauvres.

Unité 3 (U3): Elle est caractérisée essentiellement par une junipéraie rouge. En fonction de l'exposition, elle a été subdivisée en $\mathrm{U} 3 \mathrm{~A}$ et $\mathrm{U} 3 \mathrm{~B}$.

Unité 4 (U4): Elle regroupe les steppes et est subdivisée en fonction du gradient climatique latéral en U4A et U4B.

Les différentes unités géographiques du massif sont de très inégale importance. Leurs richesses en espèces varient de $10 \%$ à $42 \%$ pour les unités et de $4 \%$ à $30 \%$ pour les sousunités. Ces proportions sont fonction des étages bioclimatiques et des gradients climatiques repérés dans le massif. U2 sous bioclimat $\mathrm{SH}$ est la plus riche en espèces. A l'intérieur de U2, U2A située à l'ouest est plus riche (28\%) que U2D située à l'est (tab. 2). 


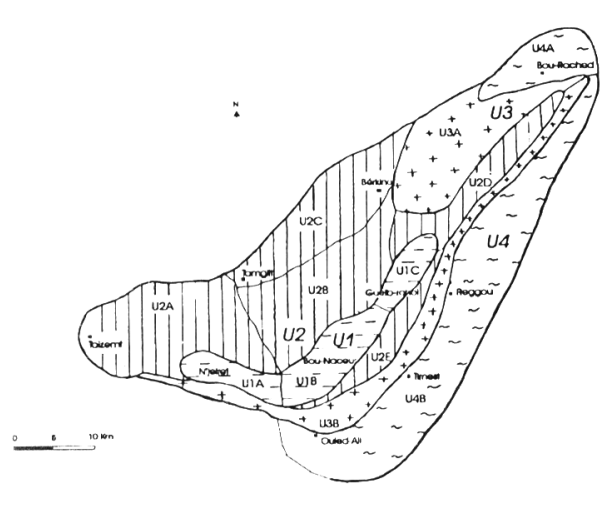

Figure 2. Unités et sous-unités géographiques de Bou-Naceur.

Sur le versant sud et en basse altitude que U2E, U3B possède un maximum d'espèces (30\%). Cette sous-unité est la plus hétérogène. Elle est dominée par une junipéraie rouge, une buxaie et localement par une chênaie verte. Nous signalons que dans cette sous-unité les cours d'eau offrent un cortège floristique important. Enfin, l'unité la plus sèche U4 est car̃actérisée par des formations steppiques nettement pauvres en espèces.

\section{Répartition des espèces dans le massif}

En analysant la répartition des espèces dans les unités et sous-unités du massif, d'après nos relevés floristiques, nous avons déterminé deux types d'aires: Aire large: Espèces récoltées dans plus de quatre sous unités. Aire restreinte: Espèces présentes dans une seule sous unité.

Pour repérer les affinités floristiques entre les différentes sous-unités, nous avons regroupé les espèces ayant les mêmes aires de répartition. Ainsi on note que cinq espèces se répartissent dans presque toutes les sous-unités de U1, U2 et U3. Ce sont par ordre d'abondance : Helianthemum croceum, Veronica rosea,
Helianthemum cinereum, Scorzonera pygmaea et Capsella bursa-pastoris. Une meilleure affinité s'observe entre les unités U1 et U2 surtout avec U2A, U2B et U2E. Par ailleurs, $\mathrm{U} 1$ et U4 sont bien distinctes l'une de l'autre puisqu'aucune espèce du massif ne leur est commune.

Parmi les espèces à large répartition dans U2 et U3, nous citons: Helianthemum pergamaceum, Bellis silvestris, Quercus ilex et Dactylis glomerata.

Certaines espèces réparties dans U4 sont signalées aussi dans U2. Il s'agit de : Agrostis verticillata, Stipa parviflora, Sanguisorba ancistroides et Rosmarinus officinalis.

\section{Endémisme}

Les taxons endémiques sont fréquents sur les hauts sommets du massif, spécialement sur l'adrar Bou-Naceur (U1B). Si on se réfère au nombre d'endémiques des unités U1, U2, U3 et U4 par rapport à leurs richesses floristiques, on trouve respectivement $40 \%$, $18 \%, 5 \%$ et $0 \%$ (tab. 3 ).

Quezel (1957) a récolté 5 taxons spéciaux au Bou-Naceur; il s'agit de: Koeleria embergeri, Phyteuma charmelii var. mesatlantica, Aster pujosii, Minuartia mairei et Calamagrostis argentea subsp. mesatlantica.

\section{Analyse biogéographique}

Parmi les espèces présentes au BouNaceur, plusieurs ont une aire de répartition méditerranéenne. Pour étudier la répartition des espèces du massif à l'extérieur du Maroc, nous nous sommes basés sur les renseignements fournis par le catalogue des plantes du Maroc (CPM), les premiers tomes de Med-Checklist et la Nouvelle Flore de l'Algérie (NFA). Nous avons trouvé ainsi que presque la totalité des espèces sont holarctiques dont $62 \%$ sont des endémiques méditerranéennes. $9 \%$ des espèces se rencontrent en Macaronésie, $7 \%$ en Eurasie et $2 \%$ seulement en Afrique.

Le massif du Bou-Naceur se rapproche 


\begin{tabular}{|c|c|c|c|c|c|}
\hline Unités & Nombre d'espèces & $\%$ & Sous unités & Nombre d'espèces & $\%$ \\
\hline \multirow{3}{*}{ U1 } & \multirow{3}{*}{139} & \multirow{3}{*}{24} & U1A & 38 & 7 \\
\hline & & & U1B & 130 & 24 \\
\hline & & & $\mathrm{U} 1 \mathrm{C}$ & 60 & 11 \\
\hline \multirow{5}{*}{$\mathrm{U} 2$} & \multirow{5}{*}{234} & \multirow{5}{*}{42} & $\mathrm{U} 2 \mathrm{~A}$ & 156 & 28 \\
\hline & & & $\mathrm{U} 2 \mathrm{~B}$ & 133 & 24 \\
\hline & & & $\mathrm{U} 2 \mathrm{C}$ & 108 & 19 \\
\hline & & & $\mathrm{U} 2 \mathrm{D}$ & 20 & 4 \\
\hline & & & & 88 & 16 \\
\hline \multirow{3}{*}{ U3 } & \multirow{3}{*}{210} & \multirow{3}{*}{35} & $\mathrm{U} 2 \mathrm{E}$ & & \\
\hline & & & $\mathrm{U} 3 \mathrm{~A}$ & 98 & 18 \\
\hline & & & U3B & 168 & 30 \\
\hline \multirow[t]{2}{*}{$\mathrm{U} 4$} & \multirow[t]{2}{*}{58} & \multirow[t]{2}{*}{10} & $\mathrm{U} 4 \mathrm{~A}$ & 46 & 8 \\
\hline & & & $\mathrm{U} 4 \mathrm{~B}$ & 41 & 7 \\
\hline
\end{tabular}

Tableau 2. Richesse floristique des unités et sous unités du massif.

beaucoup du Haut-Atlas (HA). En effet environ $80 \%$ de notre inventaire floristique est commun aux deux régions. Cela est dû certainement à la similitude de l'orographie et du climat qui ont créé les mêmes conditions pour l'épanouissement de plusieurs espèces. L'affinité du massif avec les autres régions du pays est moins évidente: $62 \%$ avec le Rif (R), $49 \%$ avec le Maroc atlantique nord (Man), $43 \%$ avec les Monts du Maroc oriental (Om) et l'Anti-Atlas (AA), $37 \%$ avec le Maroc atlantique moyen (Mam), 33\% avec les plateaux du Maroc oriental (Op), $23 \%$ avec l'Atlas saharian (As), 12\% avec le Maroc saharien (Ms) et $1 \%$ seulement avec le littoral méditerranéen (LM).

Par ailleurs, nous pouvons ajouter qu'environ $50 \%$ des espèces présentes dans le massif, le sont aussi dans 4 à 7 divisions du Maroc. $21 \%$ ont une aire très vaste englobant 8 à 11 divisions contre $36 \%$ d'espèces qui se cantonnent dans une à trois divisions seulement.

Parmi les espèces à large répartition dans le massif et au Maroc, nous citons: Stipa tenacissima, Dactylis glomerata, Polycnemum fontanesii, Arenaria serpyllifolia,
Helianthemum pergamaceum, Helianthemum croceum et Helianthemum cinereum.

D'autres espèces, au contraire, se répartissent largement dans le Bou-Naceur mais à aire restreinte ailleurs, nous citons: Cerastium gibraltaricum, Pimpinella tragium, Berberis hispanica, Alyssum spinosum, Prunus prostrata, Artemisia flahaultii et Carduncellus pomelianus.

L'étude de la répartition des taxons endémiques montre aussi, comme pour la flore totale, que le massif se rapproche beaucoup plus du Haut-Atlas (63\%) que des autres divisions géographiques du Maroc tels que l'Anti-Atlas (14\%), le Rif (12\%) ou les monts de l'oriental $(4 \%)$.

\section{CONCLUSION}

L'inventaire floristique du Bou-Naceur actuellement évalué à 557 espèces et sousespèces paraît bien important eu égard à la surface trop restreinte du massif. A ce nombre, il faut ajouter une vingtaine d'échantillons que nous n'avons pas encore pu déterminer jusqu'à 


\begin{tabular}{|c|c|c|c|c|c|}
\hline Unités & $\begin{array}{c}\text { Nombre } \\
\text { d'endémiques }\end{array}$ & $\%$ & Sous unités & Nombre d'endémiques & $\%$ \\
\hline & & & U1 A & 12 & 13 \\
\hline \multirow[t]{4}{*}{ U1 } & 56 & 60 & U1B & 56 & 60 \\
\hline & & & $\mathrm{U1C}$ & 23 & 25 \\
\hline & & & $\mathrm{U} 2 \mathrm{~A}$ & 23 & 25 \\
\hline & & & $\mathrm{U} 2 \mathrm{~B}$ & 20 & 21 \\
\hline \multirow[t]{3}{*}{$\mathrm{U} 2$} & 43 & 46 & $\mathrm{U} 2 \mathrm{C}$ & 6 & 6 \\
\hline & & & $\mathrm{U} 2 \mathrm{D}$ & 1 & 1 \\
\hline & & & $\mathrm{U} 2 \mathrm{E}$ & 14 & 15 \\
\hline \multirow[t]{2}{*}{$\mathrm{U} 3$} & 12 & 13 & $\mathrm{U} 3 \mathrm{~A}$ & 1 & 1 \\
\hline & & & $\mathrm{U} 3 \mathrm{~B}$ & 11 & 12 \\
\hline \multirow[t]{2}{*}{$\mathrm{U} 4$} & 0 & 0 & $\mathrm{U} 4 \mathrm{~A}$ & 0 & 0 \\
\hline & & & U4B & 0 & 0 \\
\hline
\end{tabular}

Tableau 3. Richesse des unités et sous unités en taxons endémiques; (les pourcentages sont calculés par rapport au nombre total d'endémiques du massif)

présent. Cependant, cet inventaire n'est pas totalement complet. L'existence d'espèces, absentes de nos relevés mais signalés précédemment par Quezel (1957), en est une preuve. Les difficultés rencontrées sur le terrain (relief très accidenté, accès difficile, pistes mal ou non entretenues, absence de véhicule approprié, neige, etc.) ont réduit considérablement l'opération d'échantillonnage dans le temps et dans l'espace, il nous a été parfois impossible d'effectuer des relevés dans certaines zones de la région.

L'étude des gradients d'humidité et la description de la végétation ont été très utiles pour découper le massif en unités floristicoécologiques supposées homogènes afin d'établir un schéma biogéographique au sein du Bou-Naceur. U1 située dans un étage de haute montagne abrite $24 \%$ de l'inventaire floristique, U2 à bioclimat subhumide possède $42 \%$, U3 à bioclimat semi-aride présente $35 \%$ et enfin $10 \%$ dans l'aride U4.

A l'échelle du Maroc, la répartition des espèces s'avère influencée par l'orographie. Ainsi les grandes ressemblances d'ordre physiographique entre le Bou-Naceur et le Haut-Atlas se traduisent par des affinités floristiques très étroites par comparaison avec les autres régions. A échelle plus large, la flore du Bou-Naceur s'affiche presque totalement holarctique avec une dominance très nette de la composante méditerranéenne.

\section{ANNEXE}

\section{Catalogue floristique du Bou-Naceur}

Pour chache espèce, il est donné; le nom scientifique et l'auteur, les synonyme dans CPM, NFA et FAN, le type biologique, les étages bioclimatiques, les sous-unités du Bou-Naceur et les divisions géographiques du Maroc. La classification adoptée dans ce catalogue est celle du CPM.

Abréviations utilisées:

$(+)$ : Espèce nouvelle pour le Moyen-Atlas (MA), (-) : Indiquée dans MA mais absente dans les relevés, $\left.{ }^{*}\right)$ : endémique marocaine.

-Types biologiques: $\mathrm{Ph}$ (phanérophyte), $\mathrm{Nph}$ (nanophanérophyte), Ch (chaméphyte), Hém (hémicryptophyte), Th (thérophyte) et $\mathrm{G}$ (géophyte) -Etages bioclimatiques: A (aride), SA (semi-aride), SH (subhumide), H (humide) et HM (haute montagne).

-Sous-unités du Bou-Naceur: (fig.2).

-Divisions géographiques du Maroc: (Fennane et al., 1999) : LM (littoral méditerranéen), Om (monts 
de l'oriental), Op (plaines et plateaux du Maroc oriental), Ms (Maroc saharien), Man (Maroc atlantique nord), Mam (Maroc atlantique moyen), $\mathrm{R}$ (Rif), MA (Moyen-Atlas), HA (Haut-Atlas), AA (Anti-Atlas), As (Atlas saharien).

\section{POLYPODIACEAE}

1. (-) Asplenium viride Huds.

Hém - HM - U1B U1C - MA HA

2. (-) Asplenium selosii Leybold

G - HM - U1B - R MA HA

\section{EQUISETACEAE}

3. Equisetum ramosissimum Desf.

G - SA - U2C U3B - LM Om Op R Man Mam MA HA As

\section{PINACEAE}

4. Pinus halepensis Mill. $\mathrm{Ph}$ - SA SH - U2C U3A U3B - Om MA HA As

5. Pinus pinaster Aiton. subsp. hamiltonii (Ten) Huguet del Villar

$$
=\text { Pinus pinaster Soland. (CPM) }
$$

$\mathrm{Ph}$ - SA SH - U2A U2B U3B - R MA

6. Cedrus libani A.Richard subsp. atlantica (Endl) Batt. \& Trabut

$=$ Cedrus libanotica Trew subsp. atlantica

(Manetti) Holmb (CPM)

$\mathrm{Ph}-\mathrm{SH} \mathrm{H}-\mathrm{U} 2 \mathrm{~A}$ U2B U2E - R MA HA

\section{CUPRESSACEAE}

7. Tetraclinis articulata (Vahl) Masters $=$ Callitris articulata (Vahl.) Link. (CPM)

$\mathrm{Ph}$ - SA - U3A - Om Man Mam R MA HA AA

8. Juniperus oxycedrus L. subsp. oxycedrus =Juniperus oxycedrus L. subsp. rufescens Asch. ( CPM)

Ph Nph - SA SH - U1A U1B U2A U2B U2C $\mathrm{U} 2 \mathrm{D}$

Om Man R MA HA As

9. Juniperus communis L. subsp. hemisphaerica (C.Presl.) Nyman

= Juniperus communis L. var. hemisphaerica

(Presl) (CPM)

$\mathrm{Ph}$ - HM - U1B - R MA HA

10. Juniperus phoenicea $\mathrm{L}$.

$\mathrm{Ph}$ - SA - U2C U3A U3B - LM Om Man Mam

R MA HA As

11. Juniperus thurifera $\mathrm{L}$.

$\mathrm{Ph}$ - SH HM - U1A U1B U1C U2B - MA HA
EPHEDRACEAE

12. Ephedra nebrodensis Goss. (M.Chl)

=Ephedra major Host. (CPM)

Nph - SH - U2B U2E - R MA HA AA

\section{GRAMINEAE}

13. (+) Pennisetum dichotomum (Forsk) Delile

Ch - A SA - U4A U4B - Ms

14. Stipa tenacissima $\mathrm{L}$.

Hém - A SA - U2E U3B U4A U4B - Om Op

Man R MA HA AA As

15. Stipa pennata L. subsp. mediterranea (Trin. \& Rupr.) Asch. \& Gr.

Hém - HM - U1C - MA HA

16. Stipa parviflora Desf.

Hém - A SA SH- U2A U3A U4A U4B - Tout le Maroc

17. (*) Stipa nitens Ball.

Hém - HM - U1B U1C U2E - MA HA AA

18. Stipa retorta Cavan.

Th - SA - U3A U3B U4A - Om Op Ms Man Mam R MA HA AA As

19.(-) Calamagrostis argentea (Lamk) DC.

${ }^{*}$ ) subsp. mesatlasica Quezel

Hém - U1A U1B U2A - MA oriental (Quezel)

20. Oryzopsis caerulescens (Desf.) Richter

Hém - SA SH - U2B U3B - Om Man Mam R

MA HA AA As

21. Polypogon monspeliensis (L.) Desf.

Th - SA - U3B - LM Om Op Ms Man Mam R

MA HA As

22. Agrostis verticillata Vill.

Hém - SA SH - U2A U3B U4A - Tout le Maroc

23. Avena sterilis L. subsp. macrocarpa (Moench) Briq.

Th - A SA - U3B U4A - LM Om Op Man Mam

R MA HA AA As

24. Avena montana Vill. Hém - HM - U1B U1C - MA HA

25. (*) Avena jahandiezii Litt.

Hém - SH - U2A U2B U2E - MA

26. Avena bromoides Gouan.

Hém - SA - U3B - Om Man R MA HA

27. Avena pratensis $\mathrm{L}$. subsp. iberica $\mathrm{St}-\mathrm{Y}$.

Hém - SH H - U1B U2A - MA

28. Avena filifolia Lag.

Hém - SH - U2E - MA As

29. Cynodon dactylon (L.) Pers.

G - A SA - U3A U4A U4B - Om Op Ms Man Mam R MA HA AA As 
30. Echinaria capitata (L.) Desf.

Th - SA SH - U2B U3B - Om Op Man Mam R MA HA AA As

31. Phragmites communis Trin.

Hy SA - U2C U3B - LM Man Mam R MA HA As

32. Koeleria phleoides (Vill.) Pers.

Th - A SA SH - U2B U4A - Op Man Mam R MA HA AA As

33. (*-) Koeleria embergeri Quezel

Hém - HM - U1B - MA

34. Koeleria splendens Presl. subsp. caudata (Link.) Stend.

Hém - SA SH - U2C U3B - R MA HA AA

35. Melica ciliata L.

Hém - SH - U2B - LM Om Op Man Mam R MA

HA AA

36. Melica cupani Goss.

Hém - SH - U2A U2B U2E - Mam R MA HA

37. Dactylis glomerata $\mathrm{L}$.

Hém - SA SH H - U2A U2B U2D U2E - LM

Om Op Man Mam R MA HA AA As

38. Cynosurus elegans Desf. subsp. obliquatus (Link.) Trab.

Th - SH H - U2A U2B U2C - Om Man R MA HA

39. Schismus barbatus (L.) Thel. subsp. calycinus

(L.) Maire \& Weiller

Th - A SA - U3A U4A U4B - Op Ms Man Mam R MA HA

40. Poa bulbosa L.

Hém - SA SH H - U2A U2B U3A - Om Op Man

Mam R MA HA AA As

41. Festuca ovina L. subsp. ovina (L.) J.D.Hook. Hém - SH HM - U1A U1B U1C U2B R MA

42. Festuca hystrix Boiss.

Hém - HM - UIB - UIC - R MA HA

43. Festuca deserti Coss. et DR. (*) subsp. maroccana Trab.

Hém - SH H HM - U 1 A U1B U IC U2A MA HA

44. Festuca elatior L. subsp. arundinacea (Schreb.) Hack.

Hém - SH - U1C U2B U2C - Op Man Mam R MA HA

45. Scleropoa rigida (L.) Gris.

Th - SA SH - U2B U3A - LM Om Man Mam R

MA HA AA

46. Bromus madritensis $\mathrm{L}$.

Th - SA U3A - LM Om Op Man Mam R MA

47. Bromus tectorum $\mathrm{L}$.

Th - SH - U2A U2B - Om Man R MA HA AA As

48. Bromus rubens $\mathrm{L}$.
Th - SA U3A U3B - LM Om Op Ms Man Mam R MA HA AA As

49. Bromus squarrosus $L$.

Th - SH H - U2A U2B - R MA HA AA As

50. Brachypodium ramosum (L.) R. \& Sch.

Hém - SA U2C U3A U3B - Om R MA HA

51. Brachypodium distachyum (L.) R. \& Sch.

Th - SA SH U2C U2E - Om Op Man Mam R

MA HA AA As

52. Lolium rigidum Gaud.

Th - SH - U2B - LM Om Op Man Mam R MA HA AA As

53. Dasypirum hordeaceum (Coss. \& Dur.) Maire = Haynaldia hordeacea (Coss. \& Dur.) Hack. (CPM)

Hém - SH - U1B - R MA HA AA As

54. Aegilops ovata $\mathrm{L}$.

Th - SH - U2B U2C U2E - Om Op Man Mam

R MA HA AA As

55. Hordeum murinum L. subsp. leporinum (Link) Asch. \& Gr.

Th - SA SH - U2B U2C U3A - Om Op Ms Man Mam R MA HA AA As

\section{CYPERACEAE}

56. (+) Cyperus laevigatus L. subsp. distachyus (All.) Maire \& Weiller

Hém - SA U3B - Man Mam HA

57. Carex halleriana Asso.

Hém - SH - U2A - Om Man Mam R MA HA AA

58. Carex hispida Willd.

G - SA - U2C U3B - LM Om Man Mam R MA

HA

59. Carex mairei Coss. \& Germ.

Hém - SH - U2B - MA

JUNCACEAE

60. Juncus maritimus Lamk.

Hém - A SA - U4B - LM Om Op Ms Man Mam R MA HA

61. Juncus bufonius L. subsp. bufonius

Th - SA - U3B - LM Om Op Ms Man Mam R MA HA AA As

LILIACEAE

62. Colchicum triphyllum Kunze.

G - SH - U2A - Op MA HA

63. Asphodelus microcarpus Viv.

G - SA - U3A U3B - Op Man Mam R MA HA AA As 
64. Allium moly L. subsp. massaesyllum Batt. \& Trab. G - SH - U2A - R MA

65. Urginea maritima (L.) Baker

G - A SA - U3A U4A - Om Op Man Mam R MA

66. Muscari comosum (L.) Miller

G - SH - U2A U2B U2C -Om Op Man Mam R

MA HA AA As

67. Asparagus stipularis Forsk.

G - A SA - U3A U4B - LM Om Op Ms Man Mam R MA HA AA As

68. Asparagus albus $\mathrm{L}$.

Nph - A SA - U3A U4A U4B - LM Om Op Man Mam R MA HA AA

69. Smilax aspera $\mathrm{L}$.

$\mathrm{Ph}$ - SA - U2C - Om Man Mam R MA HA AA As

IRIDACEAE

70. Gladiolus byzantinus Miller.

G - SA - U3A - Om Op Man Mam R MA HA AA

\section{ORCHIDACEAE}

71. Orchis coriophora L. subsp. major Maire

G - SH - U2A U2B - Man Mam MA HA

\section{SALICACEAE}

72. Populus nigra L. subsp. neopolitana (Ten) Asch. \& Gr.

Ph - SA - U2C U4B - Om Man R MA HA AA

73. Salix atrocinerea L. subsp. catalanica (Sennen) Goerz.

$\mathrm{Ph}-\mathrm{SA}$ - U3B - Man R MA HA AA

\section{FAGACEAE}

74. Quercus ilex L.

$\mathrm{Ph}$ - SA SH H - U2A U2B U2E U2D - Om Man

R MA HA AA As

\section{URTICACEAE}

75. Urtica pilulifera $\mathrm{L}$.

Th - SA - U3B - Om Op Man Mam R MA HA

\section{LORANTHACEAE}

76. Arceuthobium oxycedri M. Bieb.

Ch P - SH - U2A - Om Man R MA HA

77. Viscum cruciatum Sieb.

Ch P - SA SH - U2A - Op Man R MA HA

SANTALACEAE

78. Thesium humifusum D.C. subsp. divaricatum (Jah.) Maire
Ch P - SA SH - U2A U3B - Om Man R MA HA

\section{CYTINACEAE}

79. Cytinus hypocistis L. subsp. kermesinus (Guss.) Wettst.

G P - SA - U3A U4A - Om Man R MA HA AA

\section{POLYGONACEAE}

80. Persicaria lapathifolia (L.) S.F.Gray.

= Polygonum lapathifolium L. (CPM)

Th - A SA - U3B U4A - LM Om Man Mam R MA HA

\section{CHENOPODIACEAE}

81. Polycnemum fontanesii Dur. \& Moq.

Ch - SA SH - U2B U2C U2E U3A U3B - Om

Op Man R MA HA AA As

82. Beta maritima $\mathrm{L}$.

= Beta vulgaris L. var. maritima (L.) Koch

Th - SA - U3B - Op Om R MA HA AA As

83. Chenopodium vulvaria $\mathrm{L}$.

Th - SA - U3B - Om Op Man Mam R MA HA AA

84. Chenopodium murale L.

Th - A SA - U4A - Op Ms Man Mam R MA HA AA As

85. Chenopodium album $\mathrm{L}$.

Th - SA - U2C - Op Ms Man Mam R MA HA AA

86. Chenopodium virgatum $\mathrm{L}$

= Chenopodium foliosum (Moench) Asch. (FAN)

Th - SA SH - U2E U3A U3B - Op MA HA AA As

87. (+) Noaea mucronata (Forsk.) Asch. \& Schw. Ch - A SA - U3A U3B U4A - Om Op R HA As

\section{CARYOPHYLLACEAE}

88. Stellaria media (L.) Vill.

Th - SA - U3A U3B - Om Op Man Mam R MA HA AA As

89. Cerastium perfoliatum $\mathrm{L}$.

Th - SH H- U2A - MA

90. Cerastium gracile Dufour.

= Cerastium Riaei Desmoul. subsp. echinulatum (Coss. Dr.) Maire (CPM)

$=$ Cerastium hirtellum Pomel (FAN et NFA)

Th - SA SH -U2A U3A - Om MA HA AA As

91. Cerastium atlanticum DR.

Th - SA - U3B - R MA HA 
92. Cerastium gibraltaricum Boiss.

Ch - SH H HM - U1A U1B U1C U2A U2B U2E

- R MA HA

93. Buffonia tenuifolia $\mathrm{L}$.

Th - A SA - U3A U3B U4B - Om Op Man R MA HA

94. Minuartia hamata (Haus. \& Born.) Mattf.

$=$ Queria hispanica $\mathrm{L}$. (CPM)

Th - SH U2B U2E - Om MA HA

95. Minuartia verna (L.) Hiem.

Hém - HM - UlA U1B UIC - MA HA

96. Minuartia hybrida (Vill.) Siskin

$=$ Minuartia tenuifolia $(\mathrm{L}$.) Hiern. (CPM)

Th - SA U3A - U3B - Om Op Ms Man Mam R

MA HA AA As

97. Minuartia montana $\mathrm{L}$.

Th - A SA - U3A U4A U4B - Om Op Man Mam

R MA HA As

98. (*-) Minuartia mairei Quezel

Th - H M - U1B - MA ( Mont Bou-Naceur)

99. Arenaria serpyllifolia $\mathrm{L}$.

Th - SA SH H - U2A U2B U2C U2E U3 - Op

Man Mam R MA HA AA As

100. (+) Arenaria modesta L. Dufour

Th - A SA - U3B U4A U4B - R

101. Arenaria grandiflora $\mathrm{L}$.

Ch - SH HM - U1B U1C U2B - MA HA

102. Arenaria armerina Bory.

$\mathrm{Ch}-\mathrm{SH}-\mathrm{U} 2 \mathrm{~A}-\mathrm{R} \mathrm{MA}$

103. Arenaria pungens Lag. $(*)$ subsp. boissieri

Emb.

Ch - HM - U1B U1C - MA HA

104. (+) Spergularia diandra (Goss.) Boiss.

= Spergula diandra (Guss) Murb. (CPM)

Th - SA - U3B - Op Ms Man Mam R HA AA

105. Telephium imperati $\mathrm{L}$.

Hém - SH - U2B U2C U2E - Om Op Man Mam

R MA HA AA As

106. Polycarpon tetraphyllum $\mathrm{L}$.

Th - SA SH - U2C U3B - LM Om Man Mam R MA HA AA

107. Polycarpon polycarpoides (Biv.) Jah. \& Maire subsp. herniarioides (Ball.) Maire \& Weill.

Hém - SH HM - U1B U2A U2B U2C U2E - Om

Op R MA HA AA As

108. Paronychia argentea Lamk.

Hém - SA - U3A U3B - Op Man R MA HA

109. Paronychia kapela (Hacq.) A. Kerner. subsp. pseudo-aretioides (Emb.\& Maire) Maire

Hém - HM - UIB UIC - MA
110. Paronychia capitata (L.) Lamk.

Hém - SA SH - U2A U2C U2E - Om Op Man

R MA HA AA

111. (+) Herniaria hirsuta L. Hém - A SA - U4A U4B - LM Man Mam

112. Herniaria regnieri $\mathrm{Br}$. Bl. \& Maire = Herniaria incana Lamk. subsp. Regnieri

Br.Bl. \& Maire (CPM)

Ch - SA - U3A U3B - R MA

113. Silene vulgaris (Moench) Garcke. subsp. angustifolia Hayek.

= Silene Cucubalus Wibel (FAN)

G - SH H - U2A U2E - Om Op Man Mam R A

HA AA As

114. Silene tridentata Desf.

Th - U3A U3B U4A- A SA- Om Op Man Mam

R MA HA AA As

115. (*) Silene corrugata Ball.

Th - SA -U3B - Man Mam MA HA AA

116. (*) Silene ayachica Humb.

G - SH HM - U1A U1B U2B- MA HA

117. (*) Silene mesatlantica Maire Hém - HM - UlB - MA HA

118. Silene rubella $\mathrm{L}$. Th - A SA - U3B U4A - Om Op Man Mam R

MA HA AA As

119. Silene muscipula $\mathrm{L}$.

Th - SH - U2A U2C - Op Man Mam R MA HA

AA

120. Silene patula Desf

= Silene italica $(\mathrm{L}$.$) Pers. (CPM)$

Hém - SH - U2A U2C - Om Man R MA HA AA

121. Silene andryalifolia Pomel

= Silene mollissima (L.) Pers. subsp. velutina

(Pourr.) Maire (CPM)

Hém - SH H - U2A U2E - R MA

122. Vaccaria hispanica (Miller) Rauschert

=Vaccaria pyramidata Medik. (CPM)

Hém - SH - U2C - LM Om Op Man Mam R MA HA

AA

123. Dianthus lusitanus Brot.

Ch - SA SH - U2A U3A U3B - Op Man Mam

R MA HA AA As

RANUNCULACEAE

124. Delphinium ambiguum $\mathrm{L}$

$=$ Delphinium peregrinum $\mathrm{L}$. (CPM)

Hém - SH H - U2A U2B - Man Mam R MA HA

125. Delphinium balansae Boiss. \& Reuter

Hém - SH - U1B U2A U2C - R MA HA AA As 
126. Ranunculus aurasiacus Pomel

= Ranunculus geraniifolius subsp. aurasiacus

(Pomel) Maire (CPM)

= Ranunculus montanus subsp. aurasiacus

(Pomel) Maire (FAN)

$(*)$ var. mesatlanticus Maire

Hém - HM - UlB - MA HA

127. Ranunculus bullatus L.

Hém - SA SH - U2C U4B - LM Op Man Mam MA HA AA

128. Ranunculus spicatus Desf. subsp. maroccanus

(Coss.) Greuter \& Beurdet

= Ranunculus spicatus Desf. subsp. rupester

(Guss.) Maire (CPM)

Hém - SH - U2C - Om Man R MA HA

129. Adonis aestivalis L. subsp. squarrosa (DC)N. Th - SH - U2C - LM Om Op Man Mam R MA

HA AA

130. Adonis microcarpa $\mathrm{DC}$

=Adonis dentata Del. subsp. microcarpa (DC.)

Riedl. (CPM)

Hém - A SA - U3A U4A U4B - Op Ms Man Mam R

MA HA AA

\section{BERBERIDACEAE}

131. Berberis hispanica Boiss. \& Reuter

Nph - SH H HM - U1A U1B U1C U2A U2B

U2E - R MA HA

\section{PAPAVERACEAE}

132. Hypecoum pendulum $\mathrm{L}$.

Th - SH - U2C - Om Op Ms Mam R MA HA AA

133. Papaver rhoeas $\mathrm{L}$.

Th - SA SH - U2C U3B - LM Om Op Ms Man

Mam R MA HA AA

\section{FUMARIACEAE}

134. Platycapnos spicatus (L.) Bernh. Th - SA - U3A - Om Op Man R MA

135. Fumaria officinalis $\mathrm{L}$.

Th - SH - U2B - LM Om Op Man Mam R MA HA AA

\section{CRUCIFERAE}

136. Lepidium hirtum (L.) subsp. dhayense (Munby) Thell.

Hém - SH - U2E - Om R MA HA AA

137. Lepidium hirtum (L.) Sm. (*) subsp. atlanticum (Ball.) Maire Hém - SH H - U2A U2B - R MA HA
138. (*) Biscutella atlantica (Maire) Greuter \& Burdet

= Biscutella laevigata L. subsp. montana

(Cav.)Maire var. atlantica Maire (CPM)

Hém - SH H - U2A - R MA

139. (+) Iberis sempervirens $L$.

$\mathrm{Ch}-\mathrm{SH} \mathrm{H}-\mathrm{U} 2 \mathrm{~A}$ U2C - HA

140. Iberis atlantica (Litt. \& Maire) Greuter \& Burdet

= Iberis ciliata All. subsp. taurica (DC.) Maire (CPM)

$=$ Iberis linifolia $\mathrm{L} .(\mathrm{FAN})$

Hém - SH - U2A - Om Man MA HA As

141.Aethionema saxatile (L.) R. Br. subsp. ovalifolium (DC.)Rouy. \& Fouc

Ch - SH HM - U1B U2B - MA HA

142. Thlaspi perfoliatum L. subsp. tineoi (Paol.) Maire

Th - SH H- U2A - R MA HA AA

143. Sisymbrium runcinatum $D C$.

Th - SA - U3A U3B - Op Man Mam R MA HA

144. Sisymbrium orientale $\mathrm{L}$.

Th - SH - U2B - R MA HA

145. Sisymbrium crassifolium Cav. (*) var. atlanticum Maire

Hém - SH - U2A - Op R MA HA

146. Isatis tinctoria $\mathrm{L}$.

Hém - SH H - U2A U2C U2E - MA HA AA

147. Carrichtera annua L.DC

$=$ Vella annua $\mathrm{L}$. ( NFA et FAN)

Th - A SA - U4A - Om Ms Man Mam R MA HA As

148. (*) Vella mairei Humb.

= Pseudocytisus mairei (Humb.) Maire (FAN)

Ch - SH HM - U1A U1B U1C U2B - MA HA

149. Sinapis arvensis $\mathrm{L}$.

Th - SA- U2C - Man Mam R MA HA

150. (*) Erucastrum elatum (Ball.) O.E.Schulz

= Erucastrum laevigatum subsp. elatum (Ball.)

Maire \& Weill. (CPM)

Hém - SH - U2A - MA HA

151. Erucastrum leucanthum Coss. \& Dur.

Hém - SH - U2E - Op MA

152. Brassica repanda (Willd.) DC. subsp. confusa

( Emb. \& Maire) Hey wood

= Brassica saxatilis subsp. blancoana (Boiss.

\& Reuter) Maire (CPM)

Hém - HM - UIC - MA

153. Brassica repanda (Willd.) DC. subsp. silenifolia ( Emb. ) Greuter \&Beurdet

= Brassica saxatilis subsp. repanda (Willd.) 
Maire (CPM)

Hém - HM - U1B - MA HA

154. Brassica gravinae Ten.

Hém - SH - U2E U3B - MA HA As

155. (+) Brassica nigra (L.) Koch.

Th - SH - U2C - Man R

156. Brassica fruticulosa Cyr. subsp. cossoniana

(Boiss. \& Reuter) Maire

Hém - SA - U3A U4B - R MA

157. Raphanus raphanistrum $\mathrm{L}$. subsp. raphanistrum

Th - A SA - U3A U4A U4B - LM Om Op Man

Mam R MA HA AA

158. (+) Rapistrum rugosum (L.) Al.

Th - A SA - U4A - LM Om Op Man R

159. Raffenaldia primuloides Godr.

Hém - SH H HM - U1B U2A U2B - R MA HA

160. Nasturtium officinale $R$. Beurdet

= Rorippa nasturtium-aquaticum $\mathrm{L}$. (CPM)

Hy - SA - U3B - LM Om Op Man Mam R MA

HA AA

161. Cardamine hirsuta L.

Th - SH - U2A - LM Om Man Mam R MA HA AA

162. Hornungia petraea (L.) Reichenb

$=$ Hutchinsia petraea $(\mathrm{L}.) \mathrm{R}$. Beurdet (NFA)

Th - SA SH - U2A U2B U2D U3B - Om Op Ms Man R MA HA AA

163.Capsella bursa-pastoris (L.) Medicus

Th - SA SH - U1B U2C U2E U3B - Tout le Maroc

164. Neslia apiculata Fisch. \& al.

= Neslia paniculata subsp. apiculata Fisch. (CPM)

Th - SA - U3B - Om Op Man Mam R MA HA AA As

165. (-) Draba tomentosa Vahl

= Draba tomentosa subsp. laevipes (DC.)

Maire (CPM)

Hém - HM - UlB- MA

166.(*-)Draba hederifolia Coss. subsp. cossonii Maire Hém - HM - UIB UIC - MA HA

167. Draba hispanica Boiss.

Hém - HM - UIB UIC - R MA HA

168. Descurainia sophia (L.) Prantl

= Sisymbrium sophia L. (NFA)

Th - SH - UIB - MA HA AA As

169. Arabis alpina L. subsp. caucasica (Willd.) Briq. Ch - SH HM - UIB - R MA HA

170. Arabis pubescens (Desf.) Poiret subsp. pubescens
Hém - SH H - U2A - Om Man R MA HA

171. Arabis hirsuta (L.) Scop.

(*) var. mesatlantica Maire (CPM)

Hém - SH - U2B - MA

172. Arabis auriculata Lamk.

Th - SA SH H - U1A U2A U2B U2D U2E - Om

R MA HA AA As

173. Erysimum nervosum Pomel

=Erysimum bocconei $(\mathrm{Al}$.) Pers. (CPM)

Hém - SH H HM - U1A U1B U1C U2A U2B -

Om Man R MA HA As

174. Erysimum incanum G.Kunze.

Th - SA SH - U2A U2B U2E U3A U3B - R MA HA AA

175. (+) Erysimum repandum L.

Th - A SA - U4A - Ms

176. Alyssum linifolium Willd.

Th - SA - U3A U3B - Om Op Ms MA

177. Alyssum serpyllifolium Desf.

= Alyssum alpestre subsp. serpyllifolium (L.)

Desf. (NFA)

Ch - SH H HM - U1A U1B U1C U2A U2B U2E

- R MA HA AS

178. Alyssum granatense Boiss. \& Reuter Th - SA SH - U2A U2E U3B - Om Op Man R

MA HA AA

179. Alyssum simplex Rudolphi

= Alyssum campestre L. ( CPM )

= Alyssum parviflorum Fisch.( NFA et FAN)

Th - SA SH - U2B U3A U3B - Om Op Man

Mam R MA HA AA As

180. Alyssum spinosum $\mathrm{L}$.

Ch - SH H HM - U1A U1B U1C U2A U2B U2E

- R MA HA AA

181. Lobularia maritima (L.) Desv

= Alyssum maritimum (L.) Lamk. ( CPM)

Ch - SA-U3B - LM Om Op Man Mam R MA

$\mathrm{HA}$

182. (+) Clypeola jonthlaspi L.

Th - SA - U3A - R Man

183. (+) Notoceras bicorne Aiton.

Th - A SA - U4A U4B - Op Ms Man Mam R HA

AA

184. Matthiola fruticulosa (L.) Maire

Hém - SA - U3B - LM Om Op Man Mam R MA

HA AA

185. Matthiola perennis $\mathrm{P}$. Conti.

= Matthiola scapifera Humb. ( CPM )

Hém - HM - U1B U1C - MA HA

186. Moricandia suffruticosa (Desf.) Coss. \& Dr. 
= Moricandia arvensis subsp. suffruticosa (Desf.) Maire (CPM)

Ch - A SA - U3B U4A - Op Ms MA HA AA As

\section{RESEDACEAE}

187. (+) Reseda decursiva Forskal.

Th - SA - U3A - LM Op Ms Man R

188. (+) Reseda arabica Boiss.

Th - A SA - U4A - Op Ms

189. Reseda luteola $\mathrm{L}$

= Reseda luteola subsp. biaui (Pitard.) Maire (CPM)

Hém - SH - U2B - R MA AA

\section{CRASSULACEAE}

190. Sedum amplexicaule DC. subsp. tenuifolium

(Sm.) Greuter

$=$ Sedum tenuifolium $($ S.\& Sm.) Strobl. (CPM)

Ch - SH - U2B - Man R MA HA AA

191. Sedum teretifolium Lamk.

= Sedum album subsp. teretifolium (Lamk.)

Maire (CPM)

Ch - SH - U2A - Op Man Mam R MA HA

192. Umbilicus rupestris (Salisb.) Dandy

= Cotyledon umbilicus-veneris subsp. pendulina (DC.) Batt. (CPM)

Ch - SH - U2C - Om Man R MA

\section{SAXIFRAGACEAE}

193. (-) Saxifraga longifolia Lap. Maire subsp. pyrenaica Emb.

$\mathrm{Ch}$ - HM - U1C - MA HA

194. (*-) Saxifraga luizetiana Emb. \& Maire

Ch - HM - UlB - MA ( Bou-Naceur)

195. Saxifraga globulifera Desf.

Ch - SA - U2D - LM Om Man R MA HA

196. Ribes alpinum $\mathrm{L}$.

Nph - HM - U1B U1C - MA HA

197. Ribes uva-crispa L.

Nph - SH H HM - U1B U1C U2A U2B - R MA

HA AA

\section{ROSACEAE}

198. Cotoneaster nummularia Fisch. \& Meyer = Cotoneaster racemiflora (Desf.) Koch. (NFA)

Nph - SH H - U2A U2B U2E - R MA HA

199. (-) Amelanchier ovalis Medik.

Nph - HM - U1B - MA HA

200. Crataegus laciniata Ucria
Nph - SH - U2A U2B - Om R MA HA

201. Rubus ulmifolius Schott.

Nph - SA - U3B - LM Om Op Man Mam R MA HA AA

202. Potentilla caulescens L. subsp. petrophila Maire

$\mathrm{Ch}-\mathrm{SH}-\mathrm{U} 1 \mathrm{~B}-\mathrm{MA}$

203. Potentilla reptans $\mathrm{L}$.

Hém - SA - U2C U3B - LM Om Op Man Mam R MA HA

204. Sanguisorba minor Scop. subsp. muricata (Spach.) Briq.

Hém - SA SH - U2C - LM Man R MA HA AA

205. Sanguisorba ancistroides (Desf.) A. Beurdet Ch - SA SH - U2B U3B - Om R MA HA

206. Rosa canina L. subsp. vulgaris Sams. Nph - SA - U2C U3B - Om Man R MA HA

207. Rosa sicula Tratt.

Nph - SH H - U1B U2A - R MA HA

208. Prunus prostrata Labill.

Ch - SH HM - U1A U1B U1C U2A U2B - MA HA

209. (-) Prunus padus L.

Nph - U2B- MA ( Guelb er-Rahal)

\section{LEGUMINOSAE}

210. Genista spartioides Spach. subsp.pseudoretamoides Maire

$=$ Genista retamoides Coss. ( CPM)

Nph - SA - U2C U3A U3B - Om Op Man R MA

211. (*) Genista scorpius DC. subsp. intermedia Emb. \& Maire

Nph - SA SH - U2B U2C U2E U3A U3B - MA

212. Genista pseudopilosa Coss.

$\mathrm{Ch}-\mathrm{SH} \mathrm{H}-\mathrm{U} 2 \mathrm{~A}-\mathrm{MA}$

213. Argyrolobium zanonii (Turra) P.W.Ball. subsp. fallax (Ball.) Greuter

= Argyrolobium argenteum $\mathrm{Rchb}$. (CPM)

Ch - SA - U2C - MA HA

214. Erinacea anthyllis Link.

Ch - HM - U1A U1B U2B - MA HA AA As

215. $\left({ }^{*}+\right)$ Adenocarpus artemisiifolius Jah. \& al. $\mathrm{Nph}$ - SH - U2A U2B U2E - HA

216. Cytisus fontanesii Ball.

Nph - SA SH - U2A U2B U3A - Om R MA HA

217. Cytisus balansae (Boiss.) Ball.

$=$ Cytisus purgans $(\mathrm{L}$.) Benth. subsp. balansae

(Boiss.) Maire (CPM)

Ch - SH HM - U1A U1B U1C U2B U2E - R

MA HA AA

218. Ononis aragonensis Asso. 
Nph - SH - U2A - R MA HA

219. Ononis fruticosa $\mathrm{L}$.

Nph - SH - U1B - MA

220. (*+) Ononis atlantica Ball.

Ch - HM - UIB - HA

221. Ononis natrix L. subsp. natrix

Ch - SA SH - U2B U2E U3A - Om Man Mam R MA HA

222. Ononis spinosa L. subsp. antiquorum (L.)

Arcangeli

= Ononis antiquorum L. (CPM)

Ch - SH - U2A - R MA

223. (+) Trigonella anguina Delile

Th - A SA - U4A - Mam Ms HA

224. Medicago polycerata (L.) Trautv.

= Trigonella polycerata $\mathrm{L}$. (CPM)

Th - SA SH - U2C U3B - Om Op Man Mam R

MA HA AA As

225. Medicago suffruticosa DC. subsp. maroccana

(Batt.) Maire (CPM)

Hém - SH - U2A - R MA HA

226. Medicago truncatula Gaerth.

Th - SA - U2C - Op Man Mam R MA HA

227. Medicago doliata Carm.

= Medicago turbinata (L.) Willd (CPM)

Th - SA - U3B - Man Mam R MA AA

228. Medicago minima $\mathrm{L}$.

Th - SH - U2A - R MA HA AA

229. Melilotus elegans Ser.

Th - SH - U2B - R MA

230. Melilotus indicus (L.) Al.

Th - SA - U2C U3B - LM Om Op Man Mam R MA HA

231. Trifolium gemellum Willd.

Th - SH - U2C - Om R MA HA AA As

232. Anthyllis vulneraria $\mathrm{L}$. (*) subsp. matrisfiliae Emb. \& Maire Hém - HM - U1B - MA

233. Anthyllis polycephala Desf. Ch - SH - U2A - Om R MA

234. Lotus palustris Willd. Hém - SA - U3B - Man Mam R MA HA

235. Lotus corniculatus L. Hém - SA SH - U2A U2C - Man Mam R MA HA

236. Lotus longisiliquosus $\mathrm{R}$. de Romer

= Lotus cytisoides L. subsp. collinus (Boiss.

) Murb. (CPM)

Hém - SA - U3B - LM Om Man Mam R MA HA

237. Bituminaria bituminosa (L.) Stirton

= Psoralea bituminosa $\mathrm{L}$. (CPM)
Hém - SH - U2B - Om Man Mam R MA HA AA As 238. Astragalus armatus Willd. subsp. numidicus

(Murb.) Emb. \& Maire

Ch - SH HM - U1A U1B U2A U2B - MA HA AA As

239. Astragalus glaux $\mathrm{L}$. Hém - SH - U2C - Om Man Mam R MA HA AA

240. Astragalus bourgaeanus Coss. Hém - SH - U2A U2B - R MA HA

241. Astragalus depressus L. subsp. depressus Hém - SH - U2C - R MA HA

242. Astragalus hamosus $\mathrm{L}$.

= Astragalus hamosus L. subsp. verus Emb. \& Maire (CPM) Hém - SH - U2C - Om Man Mam R MA HA

243. (+) Astragalus reinii Ball.

Hém - SH - U2A U2C - HA

244. Astragalus caprinus L. subsp. caprinus

Hém - SASH - U2B U3B - Man Mam MA HA

AA

245. Astragalus incanus L. subsp. incanus Hém - SH - U2C - R MA HA

246. (*) Astragalus ibrahimianus Maire Ch - HM - U1B U1C - MA HA

247. Astragalus granatensis (Lamk.) Podl. = Astragalus Boissieri Fisch. (CPM)

Ch - SH H - U2A - R MA HA

248. Scorpiurus muricatus L.

= Scorpiurus sulcata L. (CPM)

Th - A SA - U3A U4A - Om Man Mam R MA

HA AA

249. Coronilla valentina L. subsp. pentaphylla

(Desf.) Batt.

Nph - SH - U2A - Man R MA HA

250. Coronilla juncea $\mathrm{L}$. subsp. pomelii Batt.

Nph - SA - U3B - Op MA As

251. Coronilla minima $\mathrm{L}$.

Ch - SH H - U1B U2A U2B - Op R MA HA

252. Coronilla scorpioides (L.) Koch.

Th - SA - U3A U4B - LM Om Op Man Mam R

MA HA AA

253. (+) Hippocrepis ciliata Willd.

= Hippocrepis multisiliquosa L. subsp. ciliata

(Willd.) Maire (CPM)

Th - SA - U2D U3A - Op Man Mam R HA AA As

254. Hippocrepis scabra DC.

Ch - SH H - U1B U2A U2B U2E - Op Man R

MA HA AA As

255. (*) Hippocrepis liouvillei Maire

= Hippocrepis Liouvillei subsp. genuina Emb. 
\& Maire (CPM)

$$
\text { Ch - HM - U1B - MA HA }
$$

256. Hedysarum confertum Desf.

= Hedysarum humile L. (CPM)

Ch - SH - U2B U2C - MA HA

257. Onobrychis argentea Boiss. subsp. cristata

(Pomel) Batt.

= Onobrychis argentea Boiss. subsp. africana

(Sirj.) Maire (CPM)

Ch - SA - U3B U4B - Op MA HA

258. Onobrychis saxatilis (L.) Lamk.

Ch - SH - U2A - MA HA AA

259. $\left({ }^{*}\right)$ Cicer atlanticum (Coss.) Maire

G - SH HM - U1B - HA

260. Vicia sativa $\mathrm{L}$. subsp. amphicarpa $\mathrm{L}$. Batt.

Th - SA - U2C - Op Man Mam R MA HA AA

261. Vicia glauca C.Presl. (*) var. mesatlantica

Emb. \& Maire

G - SH HM - U1B - MA

GERANIACEAE

262. (*) Geranium nanum Coss.

= Geranium cinereum Cav. subsp. nanum

(Coss.) Maire (CPM) Hém - HM - U1B - MA HA

263. Geranium pyrenaicum Burm. Th - SH - U2C - MA HA AA

264. Geraniumm molle L.

Th - SA SH - U2A U2C - Om Op Man Mam R

MA HA AA As

265. Geranium rotundifolium $\mathrm{L}$.

Th - SA - U3B - Om Op Man Mam R MA HA AA As

266. Erodium cicutarium (L.) L'Hérit.

Th - SA SH - U2A U2B U3A - Om Op Ms Man R MA HA AA

267. (+) Erodium ciconium (L.) L'Hérit.

Th - SA - U3A - Om Man Mam R HA

268. Erodium gutatum (Desf.) Willd.

Hém - A SA - U4A - Om Op Ms Man Mam R

MA HA AA As

269. Erodium malacoides (L.) L'Hérit.

Th - SA - U2C - Op Man Mam R MA HA AA

\section{LINACEAE}

270. Linum suffruticosum L. subsp. suffruticosum

$\left.{ }^{*}\right)$ var. matris-filiae Emb. \& Maire (CPM)

Ch - SH HM - U1B U2B U2E - Op Man R MA HA As

271. Linum austriacum (L.) subsp. gaetulum
Hém - SH - U2A - Om Op MA AA

\section{ZYGOPHYLLACEAE}

272. Peganum harmala $\mathrm{L}$.

G - A SA - U4A U4B - Ms Man Mam R MA HA AA As

273. (+) Fagonia glutinosa Delile.

Ch - A SA - U4B - Ms AA

\section{RUTACEAE}

274. Ruta montana L.

Ch - SA - U2C - Om Man Mam R MA HA AA

POLYGALACEAE

275. Polygala rupestris Pourret

$\mathrm{Ch}$ - SH - U2B - Op Ms R MA HA AA

EUPHORBIACEAE

276. Euphorbia hirsuta L.

= Euphorbia pubescens Vahl. (CPM)

Hém - SA SH - U2B U2C U3B - LM Om Op

Man Mam R MA HA AA

277. Euphorbia helioscopia L.

Th - SH - U2C - LM Om Op Man Mam R MA HA AA

278. Euphorbia nicaeensis $\mathrm{Al}$.

Ch - SH H HM - U1A U1B U2A U2B - MA HA

279. Euphorbia exigua $\mathrm{L}$

Th - SA - U3B - LM Om Op Man Mam R MA HA AA

280. (+) Euphorbia falcata L.

Th - SA - U3A - LM Om Op Man Mam R HA

281. Euphorbia sulcata Loisel

Th - SH - U2B - Om Op Man Mam R MA HA AA

282. (*) Euphorbia mazicum Emb. \& Maire

Hém - SH - U2C - MA

BUXACEAE

283. Buxus balearica Willd.

$\mathrm{Nph}$ - SH SH - U2B U2C U3B - Om Man R MA HA As

ANACARDIACEAE

284. Pistacia lentiscus L.

Nph - SA - U2D U3A U3B - Om Man Mam MA HA As

285. Pistacia terebinthus $\mathrm{L}$.

$\mathrm{Ph}$ - SA SH - U2E U3B - Om Man Mam R MA HA As 
286. Pistacia atlantica Desf.

$\mathrm{Ph}$ - SA - U2C U3B U4B - Tout le Maroc

\section{ACERACEAE}

287. Acer monspessulanum $\mathrm{L}$.

$\mathrm{Ph}-\mathrm{SH}-\mathrm{U} 2 \mathrm{~A}$ U2E -R MA HA As

\section{RHAMNACEAE}

288. Rhamnus pumila Turra.

Ch - HM - UlB - R MA HA

289. Rhamnus lycioides L. subsp. oleoides (L.)

Jah. \& Maire HA As

Nph - SA - U3A U3B - Om Man Mam R MA

290. Rhamnus lycioides L. subsp. atlantica (Murb.)

Jah. \& Maire

Ch - HM - UIB - MA HA

\section{MALVACEAE}

291. Malva hispanica $\mathrm{L}$.

Th - SA - U3B - Man Mam R MA HA

292. Malva sylvestris $\mathrm{L} .\left({ }^{*}\right)$ subsp. subacaulis

Maire

Th - SH HM - UIA UIB UIC - HA

\section{GUTTIFERAE}

293. Hypericum tomentosum L. subsp. pubescens (Boiss.) Al.

Hém - SA - U2C - LM Om Man Mam R MA HA AA

\section{CISTACEAE}

294. Cistus creticus L. subsp. eriocephalus (Viv.) Greuter \& Burdet

$=$ Cistus villosus $\mathrm{L}$. (CPM)

Ch - SA - U3B - Om Man Mam R MA HA AA

295. Cistus libanotis $\mathrm{L}$.

Ch - SA - U3A U3B - Om MA

296. Helianthemum papillare Boiss.

Th - A SA - U2C U4A-Om Op Ms R MA HA AA

297. Helianthemum pergamaceum Pomel

= Helianthemum pilosum (L.) Pers. var. pergamaceum P.M. (CPM)

Ch - SA SH HM - U1A U2B U2C U2D U2E

U3A U3B

Om Op Ms Man R MA HA AA As

298. Helianthemum croceum (Desf.) $P$.

Ch - SA SH H HM U1A U1B UIC U2A U2B

U2C U2D U2E U3A U3B
Om Op Man Mam R MA HA AA

299. Helianthemum cinereum (Cav.) Pers. subsp. rotundifolium (Dunal.) Greuter \& Burdet = subsp. rubellum (Presl.) Maire (CPM) Ch - SA SH HM - U1A U1B U2A U2E U3B Om Op Man R MA HA AA As

VIOLACEAE

300. (+) Viola odorata L Hém - SH - U2A - Man R HA

301. (*) Viola saxifraga Maire Ch - HM - U1B - MA HA

302. Viola munbyana Boiss. \& Reuter (*) var. rifana Emb. \& Maire G - SH - U1B - R MA

THYMELAEACEAE

303. Thymelaea virgata (Desf.) Endl. Nph - SA - U3B - Man R MA HA

304. Thymelaea virescens Coss. \& Dr. $\mathrm{Ch}-\mathrm{SH}$ - U2C -Om MA HA

305. Thymelaea tartonraira (L.) Al. Ch - SA - U3B - R MA

\section{LYTHRACEAE}

306. Lythrum junceum Banks \& Solan $=$ Lythrum meonanthum Stendel (CPM) Hém - SA - U3B - Om Op Man Mam R MA HA AA As

ARALIACEAE

307. Hedera helix L. subsp. canariensis (Willd) Maire

$\mathrm{Ph}-\mathrm{SH}$ - U2B - Om Man R MA HA AA

UMBELLIFERAE

308. Eryngium bourgati Gouan. Hém - SH HM - U1B U1C - R MA HA

309. Eryngium ilicifolium Lamk. Th - A SA - U3A U4B - Om Ms Man R MA HA AA As

310. Torilis arvensis (Huds.) Link. subsp. neglecta Kerner Th - SA - U2C U3B - Om Op Man Mam R MA HA

311. Caucalis bifrons (Pomel) Maire Th - SH H - U2A U2B - Om Man R MA HA AA As 312. Caucalis leptophylla $\mathrm{L}$.

Th - SH H - U2A - Om Man Mam R MA HA AA 313. Caucalis latifolia $\mathrm{L}$. 
Th - SA SH - U2C U3B - Man R MA HA

314. Orlaya platycarpa (L.) Koch.

Th - SH - U2C - Op R MA HA AA

315. Bupleurum semicompositum $\mathrm{L}$.

Th - SA - U3A - LM Om Op Man Mam R MA HA

316. Bupleurum atlanticum Mur.

Ch - SA SH H - U2A U2B U2C U2D U2E - MA

HA AA As

317. Bupleurum spinosum L.fil.

Ch - SH H HM - U1A U1B U1C U2A U2B U2E

- Om R MA HA AA

318. (+) Pituranthos scoparius (Coss. \& Dr.)

Nph - SA - U3B - Op Ms Man R HA AA As

319. Brachyapium dichotomum (L.) Maire

Th - SA - U3A U3B - Op Man Mam MA HA

AA

320. Pimpinella tragium Vill.

Hém - SH HM - U1A U1B U1C U2A U2B U2E

- R MA HA As

321. (+) Anethum graveolens $\mathrm{L}$.

Th - A SA - U3A U3B - Man R As

322. Ferula communis $\mathrm{L}$.

G - SA SH - U2B U3A U3B - Om Op Man R

MA HA AA As

323. Daucus muricatus L.

Th - SH - U2A - Om Man Mam R MA HA

324. Daucus durieva Lange

Th - SA - U3B - Om R MA HA

PRIMULACEAE

325. Primula acaulis $\mathrm{L}$.

= Primula vulgaris Huds. (CPM)

Hém - SH H - U2A - R MA

326. Androsace maxima $\mathrm{L}$.

Th - SA SH - U2A U2D U3A U3B - Op Man R MA HA AA As

327. (+) Androsace villosa L. (*) var. subexcarpa

Emb.\& Maire

Hém - HM - U1B - HA

328. Samolus valerandi $\mathrm{L}$.

Hém - SA - U3B - LM Om Ms Man Mam R MA

HA AA As

329. Asterolinum linum-stellatum (L.) Duby.

Th - SA - U2D U3A - Om Op Man Mam R MA

HA AA As

330. Anagallis arvensis $\mathrm{L}$.

= Anagallis arvensis subsp. Phoenicea Vollman (CPM)

Th - SA SH - U2A U2B U3A U3B - Om Op
Man Mam R MA HA AA

331. (+) Coris monspeliensis $\mathrm{L}$.

Ch - A SA - U4A U4B - LM Man Mam HA

PLUMBAGINACEAE

332. Armeria ebracteata Pomel

= Statice ebracteata $($ Pomel) Maire (CPM)

Hém - SH H HM - U1B U2A - Om MA

333. Limonium lobatum (L.fil.) Chaz.

= Limonium Thouini (Viv.) O.Kunze (CPM)

Th - A SA - U4B - Om Op Ms Man Mam MA

HA AA

334. (+) Limonium echioides (L.) Mill.

Th - SA - U3A - Om Op Man R

\section{OLEACEAE}

335. Fraxinus angustifolia Vahl.

$\mathrm{Ph}$ - SA SH -U2B U2C - Om Man Mam R MA

HA

336. Fraxinus dimorpha Coss. \& Dr.

= Fraxinus xanthoxyloides Wall. (CPM)

$\mathrm{Ph}$ - SH H - U2A U2B U2E - MA HA AA As

337. Phillyrea latifolia $\mathrm{L}$

= Phillyrea angustifolia L. subsp. latifolia

(L.) Maire (CPM)

Ph - SA - U3A - Man R MA HA

338. Olea europaea $\mathrm{L}$.

$\mathrm{Ph}$ - SA - U3A - Om Op Ms Man Mam R MA

HA AA As

339. Jasminum fruticans $L$.

Nph - SA SH H - U2C - LM Om Man Mam R

MA HA AA AS

GENTIANACEAE

340. Blackstonia perfoliata (L.) Huds. subsp. grandiflora Maire

Th - SA - U2C - Man R MA

APOCYNACEAE

341. Nerium oleander $\mathrm{L}$.

Nph - SA - U2C U3B - LM Om Op Ms Man

Mam R MA HA AA

ASCLEPIADACEAE

342. $(+)$ Pergularia tomentosa $\mathrm{L}$.

Ch - A SA - U4B - Ms

CONVOLVULACEAE

343. Cuscuta planiflora Ten.

= Cuscuta epithymum L. subsp. planiflora 
(Ten.) Rouy. (NFA)

Th P - SH - U2A - Om Op Ms Man Mam R MA

HA AA As

344. Convolvulus arvensis $\mathrm{L}$.

$\mathrm{G}$ - SH - U2C - Om Op Ms Man Mam R MA HA

AA As

345. Convolvulus lineatus $\mathrm{L}$.

Hém - SH - U2B - Om Op R MA HA As

346. Convolvulus cantabrica L. (*) subsp. mazicum

(Emb. \& Maire) Maire

Hém - SH - U2B U2C - R MA

347. (+) Convolvulus valentinus Cav. subsp. suffruticosus (Desf.) Maire Hém - SA - U3B - Om Op Man

348. (+) Convolvulus supinus Coss. \& Kral.

G - A SA - U3A U4A - Op Ms HA AA

\section{BORAGINACEAE}

349. Cynoglossum dioscoridis Vill.

Th - SH - U2B - R MA HA AA

350. Cynoglossum creticum Mill.

Th - SA - U2C U3B - Om Op Man Mam R MA

HA AA

351. Anchusa undulata L. subsp. undulata Hém - SH H - U2A - MA HA

352. Lappula barbata (M. Bieb.) Gurk. (*) var. brevistyla (Ball.)Brand.

Th - SH H - U2A U2B - Om R MA HA

353. Myosotis ramosissima Rochel

= Myosotis collina Hoffm. (CPM)

Th - SH H H - U2A-Om Man Mam R MA HA

AA

354. (*) Myosotis atlantica Vesterg

= Myosotis alpestris F.W. Schm. subsp.

albomarginata Maire (CPM)

Th - SH HM - UIA UIB U2A - MA HA AA

355. Lithospermum arvense $\mathrm{L}$.

Th - SA SH - U2C U3B - Om Op Man Mam R

MA HA AA As

356. Echium flavum Desf.

Hém - SH - U2B - Om Man R MA HA

357. Echium humile Desf. subsp. pycnanthum

(Pomel.) Greuter \& Burdet

Hém - SA - U3A - LM Op Man MA HA AA

358. Rochelia disperma (L.) C.Koch.

Th - SH - U2C - MA HA AA As

\section{LAMIACEAE}

359. Ajuga iva (L.) Schreb.

Hém - SH - U2C U2E - Om Op Man Mam R
MA HA AA

360. Teucrium fruticans $\mathrm{L}$.

Nph - SA - U3A U3B - LM Om Man Mam R

MA HA

361. Teucrium pseudo-chamaepitys $\mathrm{L}$.

Ch - SA - U3B - LM Om Man Mam R MA HA

362. Teucrium chamaedrys $\mathrm{L}$.

Ch - SH - U2A - R MA HA

363. $(*)$ Teucrium musimonum Humb

Ch - HM - U1B U1C - MA

364. (*) Teucrium gattefossei Emb.

Ch - SA - U3B - MA

365. Teucrium rotundifolium Schreb. subsp. rotundifolium (CPM)

Ch - SA SH - U2C U2D U3A - R MA HA AA

366. Teucrium capitatum $\mathrm{L}$.

= Teucrium Polium L. subsp. capitatum (L.)

Briq. (CPM)

Ch - SA SH - U2A U2B U3B - Man R MA HA

AA

367. (+) Teucrium aureum subsp. flavovirens

(Batt.) S. Puech

Ch - SH HM - U1B U2E - HA

368. Rosmarinus officinalis $\mathrm{L}$.

Nph - A SA SH - U2C U2D U3A U3B U4A

U4B - Om R MA HA

369. Scutellaria orientalis L. (*) var. demnatensis

(Coss.) Batt. (CPM)

$\mathrm{Ch}-\mathrm{SH}-\mathrm{U} 2 \mathrm{~A}$ U2B - MA HA

370. Marrubium vulgare $\mathrm{L}$.

Ch - SA - U2C - LM Om Op Ms Man Mam R

MA HA AA As

371. Marrubium supinum $\mathrm{L}$.

Ch - SH - U2A - Op MA As

372. (*) Marrubium ayardii Maire

Ch - SH - U2A U2B U2E - MA HA

373. (*) Marrubium multibracteatum Humb. et

Maire

Ch - HM - UIB U1C - MA HA

374. Sideritis montana $\mathrm{L}$.

Th - SA SH - U2A U3B - Om Op Man Mam R

MA HA AA As

375. Sideritis hirsuta $\mathrm{L}$.

Ch - SH H - U2A - R MA HA

376. Sideritis incana $\mathrm{L}$.

Ch - SH H HM - U1B U2A - R MA HA

377. (*) Nepeta atlantica Ball.

Hém - SH - U2A - MA HA AA

378. Nepeta amethystina Poiret

= Nepeta nepetella L. subsp. amethystina 
(Poiret) Briq. (CPM)

Hém - SH - U1A U1B U2A U2B U2E - MA HA As

379. (*) Pitardia caerulescens Maire

Ch - SA SH - U2C U2D U3B - Op MA

380. (*) Dracocephalum renati Emb.

Ch - HM - U1B U1C - MA HA

381. Lamium amplexicaule $\mathrm{L}$.

Th - SA SH - U2C U3B - LM Om Op Man Mam R MA HA AA As

382. Lamium mauritanicum Batt.

= Lamium amplexicaule L. subsp. mauritanicum (Gand.) Maire (CPM)

Th - SH - U2B - Om Man R MA HA

383. Salvia lavandulifolia $\mathrm{Vah}$. subsp. blancoana

(Webb.\& Heldr.) Ros. \& Blanca.

= Salvia aucheri Boiss. subsp. blancoana (Wb.\& Hl.) Maire (CPM)

Ch - SH - U2A U2B U2E - MA HA

384. Salvia phlomoides Asso.

Hém - SA SH - U2B U3B - Om Man MA HA

385. Salvia argentea $\mathrm{L}$. subsp. patula (Desf.) Maire Hém - SH - U2A - Om Op Man Mam R MA HA

386. Salvia verbenaca $\mathrm{L}$.

Hém - SH - U1B - Om Op Man Mam R MA HA

AA

387. Satureja grandiflora (L.) Sch. subsp. baborensis (Batt.)Briq.

$\mathrm{Ch}-\mathrm{SH}-\mathrm{MA}$

388. Satureja heterotricha (Boiss. \& Reuter)

Greuter \&Burdet

= Satureja calamintha (L.)Sch. subsp. ascendens (Jord.) Briq. (CPM)

Ch - SA - U2D U3A - Om Man Mam R MA HA

389. Satureja alpina (L.) Scheel. subsp. meridionalis

(Nyman) Greuter \& Burdet

= Satureja alpina subsp. granatensis (Boiss.

\& Reuter) Maire (CPM)

Ch - SH H - U2A U2E - Om Man R MA HA AA

390. (*) Satureja atlantica (Ball.) Maire

Ch - SH HM - U1B UIC - MA HA AA

391. (*) Origanum elongatum (Bonnet) Emb. \&

Maire

Ch - SH H - U2A U2B - R MA

392. (*) Thymus atlanticus (Ball.) Roussine

$=$ Thymus serpyllum L. subsp. atlanticus (Ball.)

Maire (CPM)

Ch - HM - UIB U1C - MA HA

393. Thymus munbyanus Boiss. \& Reuter subsp. coloratus (Boiss. \& Reuter) Greuter \& Burdet $=$ Thymus ciliatus (Df.) Ben. subsp. coloratus
(Boiss. \& Reuter)Batt. (CPM)

Ch - SA SH - U2C U2D U3A U3B - Om MA

394. Thymus munbyanus Boiss. \& Reuter

$=$ Thymus ciliatus (Df.)Ben. subsp.

munbyanus (Boiss. \& Reuter)Batt. (CPM)

Ch - SH HM - U1A U1B UIC U2A U2B - MA

395. Thymus algeriensis Boiss. \& Reuter

Ch - SA - U3B - MA HA

396. Mentha suaveolens Ehrh.

= Mentha rotundifolia L. subsp. eurotundifolia Maire (CPM)

Hém - SA - U3B - LM Om Man Mam R MA HA AA As

\section{SOLANACEAE}

397. (+) Lycium intricatum Boiss. subsp. Pujozii Sauv.

Ch - A SA - U4B - LM Om Op Man Mam R HA AA

398. Atropa baetica Willk.

Hém - SH - U2B - R MA

\section{SCROPHULARIACEAE}

399. Verbascum maurum Maire \& Murbeck

Th - SH - U2B - Man MA HA

400. Verbascum simplex Hoffm. \& Link. (*) var. gaetulum Maire

Th - SH - U2E - Om MA HA

401. Linaria tristis (L.) Mill. subsp. marginata (Desf.) Maire

Hém - SH H - U2A U2B - Om MA As

402. Linaria tristis (L.) Mill. $\left({ }^{+}\right)$subsp. lurida

(Ball.) Maire

Hém - HM - U1B - HA

403. Linaria multipunctata (Brot.) Hoffm.

Th - SH H - U2A U2E - Man Mam MA HA AA

404. (+) Linaria arvensis (L.) Desf.

Th - SA - U3B - Mam R HA AA

405. Linaria simplex (Willd.) DC.

Th - SA SH - U2A U2B U3A U3B - Om Man

R MA HA AA As

406. Linaria rubrifolia Robill. \& Cast.

Th - A SA - U3A - Om Op Man Mam R MA HA

407. Linaria origanifolia (L.) DC. subsp. flexuosa

(Desf.) Maire

Hém - SH HM - U1B U2E - Om MA HA As

408. Antirrhinum majus L. subsp. hispanicum

(Chav.) Maire

Ch - SA SH - U2E U3B - MA HA

409. Scrophularia laevigata $V$ ahl. subsp. pubescens 
Hém SH - U2A - Om R MA HA

410. Scrophularia aquatica $\mathrm{L}$.

Hém - SA U2C U3B - Op Man Mam R MA HA

411. Scrophularia canina L.

Ch - SH - U2A - Om Op Man Mam R MA HA AA As

412. Veronica hederifolia L. subsp. maura Murbeck.

Th - SH - U1B U2C - R MA HA AA As

413. Veronica rosea Desf.

Hém - SA SH H HM - UIA UIB U1C U2A $\mathrm{U} 2 \mathrm{~B}$

U2D U2E U3B - Om R MA HA AA As

414. Veronica anagallis-aquatica L. subsp. aquatica (Bernh.) Maire

Hém - SA SH - U2B U2C U3B - LM Om Op

Ms Man Mam R MA HA AA As

415. Odontites viscosa (L.) Rchb. subsp. eriopoda

Litt. \& Maire

Th - SH - U2A - MA

416. (*) Odontites mesatlantica Emb. \& Maire

Th - SH - U2A - MA

\section{OROBANCHACEAE}

417. Orobanche variegata Wallr.

G P - SH HM - U1B U2B - MA

418. Orobanche amethystea $\mathrm{L}$.

G P - SA SH - U2E U3B - MA HA

\section{GLOBULARIACEAE}

419. Globularia alypum L.

Ch - SA SH - U2C U2D U3B - Om Man R MA HA AA

420. $\left(^{*}\right)$ Globularia nainii Batt.

Ch - SA SH - U2B U2E U3B - Op MA HA

421. (*) Globularia liouvillei Jah. \& Maire

Hém - SH HM - UIB UIC - MA HA

PLANTAGINACEAE

422. Plantago lagopus $\mathrm{L}$.

Th - SA SH - U2E U3B - LM Om Op Man Mam R MA HA AA As

423. Plantago ovata Forsk.

Th - A SA - U4A - Om Op Man Ms MA HA AA

424. Plantago albicans $\mathrm{L}$.

G - A SA - U2C U3B U4A - LM Om Op Ms

Man Mam R MA HA AA

425. Plantago coronopus $\mathrm{L}$.

Th - SA SH - U3A U3B - Om Op Ms Man Mam R MA HA AA
426. Plantago cupanii Goss.

= Plantago coronopus L. subsp. cupanii

(Guss.) Nyman (CPM)

Hém - HM - U1B - Om R MA HA AA

427. Plantago afra $\mathrm{L}$.

= Plantago psyllium $\mathrm{L}$. (CPM)

Hém - SA SH - U3A U3B - Om Op Ms Man

Mam R MA HA AA As

428. Plantago mauritanica Boiss. \& Reuter Ch - SA SH - U2A U3A - MA HA

\section{RUBIACEAE}

429. Asperula arvensis $\mathrm{L}$.

Th - SH - U2C - Man Mam R MA HA

430. Asperula hirsuta Desf.

$\mathrm{Ch}$ - SA SH H - U2A U2B U2C U3B - Om Op

Man Mam R MA HA AA As

431. Galium fruticosum Willd. subsp.ephedroides

(Willk.) Pau. \& F. Q.

Ch - SA - U2D U3A - Om Ms R MA HA AA As

432. Galium pumilum Murr. (*) var. mesatlanticum

Maire

= Galium pusillum L. (NFA)

Hém - HM - UlB UIC - MA

433. (+) Galium palustre L.

$\mathrm{G}-\mathrm{SH}-\mathrm{U} 2 \mathrm{~A}-\mathrm{R}$

434. (+) Galium viscosum Vahl. subsp. eu-viscosum Maire

Th - SH - U1B - Om Man Mam R

435. Galium parisiense L. subsp. eu-parisiense Beurdet

Th - SA - U3B - Man Mam R MA HA AA

436. Galium aparine L. subsp. spurium (L.) Hartm. Th - SA SH - U2B U2E U3B - Om Mam MA

HA AA As

437. Galium tricorne Stokes

Th - SH - U2C - LM Om Man Mam R MA HA AA

438. Galium valantia Weber.

Th - SA - U2C - Om Op Man Mam R MA HA AA

439. Galium verticillatum Danth.

Th - SA - U3B - R MA HA

440. Galium murale (L.) Al.

Th - SA SH - U2B U3B - LM Om Op Man Mam R MA HA AA

441. Rubia laevis Poiret G - SH - U2A - Om Man R MA HA AA

442. Rubia peregrina $\mathrm{L}$.

Ch - SA - U3B - Om Man Mam R MA HA 
443. Callipeltis cucullaria (L.) Stev.

Th - SA - U3B - Om Op Ms Mam R MA HA AA As

\section{CAPRIFOLIACEAE}

444. Lonicera arborea Boiss. Nph - SH - U2B - Man R MA HA

445. Lonicera pyrenaica $\mathrm{L}$.

Nph - HM - UIB UIC - MA HA

\section{VALERIANACEAE}

446. Kentranthus angustifolius DC. subsp. battandieri Maire

$$
\text { Ch - SH -bU1B U2E - Om MA HA }
$$

447. Valeriana tuberosa $\mathrm{L}$.

G - SH - U2C - Om Man R MA

448. (-) Valeriana globulariifolia Ran.

G - U1B U1C - MA

\section{DIPSACACEAE}

449. Scabiosa columbaria L.

Hém - SH - U2B - MA HA

450. Lomelosia stellata (L.) Rafin

= Scabiosa stellata L. subsp. monspeliensis

(Jacq.) Rouy. (CPM)

Th - A SA - U3A U3B U4A - LM Om Op Man Mam Ms R MA HA AA As

\section{CAMPANULACEAE}

451. Campanula filicaulis Dur.

Hém - SH H HM - U1B U2A U2B U2E - Man

MA HA AA As

452. Campanula saxifragoides Doum.

Hém - SH H - U2A U2B - MA

453. Legousia falcata (Ten.) Samp.

= Specularia falcata A. DC. (NFA)

Th - SA - U3B - Om Man Mam R MA HA

454. (-) Phyteuma charmelii Vill. (*) var mesatlantica Quezel

HM - U1B - MA

455. (-) Jasione crispa (Pouret.) Samp.

= Jasione humilis Lois. (CPM)

Hém - HM - U1B - MA HA

\section{ASTERACEAE}

456. Bellis annua L. subsp. microcephala (Lange)

Batt.

Th - SA - U3A U3B - Om Op Man Mam R MA

HA AA As

457. Bellis silvestris $\mathrm{Cyr}$.
Hém - SA SH H - U2A U2B U2D U2E U3A

U3B

Om Man Mam R MA HA AA

458. $(*)$ Bellis caerulescens Coss.

Hém - HM - UlB - MA HA AA

459. (*) Erigeron celerieri Emb. \& Maire

Hém - HM - UlB - MA

460. (*-) Aster pujosii Quezel

Hém - U1B - MA

461. Micropus bombycinus Lag. Th - SA - U2D U3A U3B - Om Man Mam R MA HA AA As

462. Evax crocidion Pomel

Th - SH - U2A U2B U3B - Om MA

463. Filago germanica $\mathrm{L} .\left(^{*}\right)$ subsp. faurei Maire $\mathrm{Th}$ - SA - U3A - Om Op

464. Phagnalon saxatile (L.) Coss. subsp. eusaxatile Maire

Ch - SA - U2C - Om Man Mam R MA HA AA

465. (*-) Phagnalon embergeri Humb. \& Maire

Ch - SH - U2E - MA HA

466. Elichrysum lacteum Coss. \& Dr.

Hém - SA - U3B - MA HA

467. Elichrysum stoechas (L.) DC. subsp. rupestre (Raf.) Maire

Ch - SA - U3A U3B - LM Om Man R MA

468. (-)Elichrysum angustifolium DC.

= Elichrysum italicum (Roth.) G. Don. (NFA)

Ch - SA SH - U2E U3B - MA

469. Inula montana $\mathrm{L}$.

Hém - SH - U2A - R MA HA As

470. Pallenis spinosa (L.) Cass. subsp. cuspidata

(Pomel) Batt.

Hém - SA - U3B - Ms MA AA As

471. (+) Asteriscus maritimus (L.) Less

Ch - A SA - U4A U4B - LM Om Op Man

472. Santolina rosmarinifolia $\mathrm{L}$. subsp. pectinata

(Lag.) Maire

Ch - SA SH - U3B U2E - R MA HA As

473. $(*)$ Ormenis scariosa (Ball.) Litt. \& Maire

$\mathrm{Ch}-\mathrm{HM}$ - UlB - MA HA AA

474. Ormenis africana Jord. \& Fou.

Ch - SH - U2A - Op MA As

475. (*-) Anacyclus atlanticus Litt. \& Maire

Hém - HM - UlB - MA HA

476. Anacyclus Pyrethrum (L.) Goss.

Hém - SH - U1B U2E - Op Man MA HA AA

477. Achillea santolina $\mathrm{L}$.

Hém - SA - U3B - Op Man MA HA

478. (*) Leucanthemum maroccanum (Batt.) Maire 
Ch - SA - U3B - Mam MA HA

479. (*) Leucanthemum redieri Maire

Hém - SH HM - UIA UIB UIC - MA HA

480. Leucanthemum mairei Humb.

Hém - HM - UIB UIC - MA HA

481. (*) Leucanthemum mesatlanticum Emb. \& Maire

Hém - SH HM - U1B U1C U2B - MA

482. Chrysanthemum coronarium $\mathrm{L}$.

Th - SA - U3A U3B - LM Om Op Man Mam R MA HA AA

483. Chrysanthemum segetum $\mathrm{L}$.

Th - A SA - U4A - Om Op Man Mam R MA HA

484. (*) Artemisia mesatlantica Maire

Ch - SH HM - U1A UIB U1C U2B - MA HA

485. $\left(^{*}\right)$ Artemisia negrei Ouyahya

=Artemisia mesatlantica var. subsimplex Hum.

\& Maire (CPM)

Ch - SH - U1B U2E - MA HA

486. (*) Artemisia flahaultii Emb. \& Maire

Ch - SH HM - U1A U1B U1C U2A U2B U2E - MA

487. Artemisia herba-alba Asso.

Ch - A SA - U3A U4A - Om Op Ms Man Mam R MA HA AA As

488. (*) Hertia maroccana Maire

Nph - SA SH - U2B U2E U3B - Op Ms HA

489. Senecio vulgaris $\mathrm{L}$.

Th - SA SH - U2E U3B - LM Om Op Man Mam R MA HA AA

490. $(*+)$ Senecio chalureaui Humb.

Th - HM - UIB - HA

491. Senecio giganteus Desf.

Hy - SH - U2C - Man MA HA

492. Senecio linifolius $\mathrm{L}$

Hém - SA - U3B - Op R MA

493. (+) Calendula aegyptiaca Pers. subsp. tripterocarpa (Rupr.) Lanza.

Th - A SA - U4A - Op Ms Mam AA

494. Echinops spinosus L. subsp. bovei (Boiss.)

Mur.

Hém - A SA- U4B - Ms Mam MA HA AA

495. Xeranthemum inapertum (L.) Willd.

Th - SA - U3A - Om Man Mam R MA HA AA As

496. Carlina atlantica Pomel

Hém - SA SH - U2B U3B - Om R MA

497. Carlina involucrata Poirret

Hém - A SA SH - U2A U4A - Op Man Mam

R MA HA As
498. Atractylis humilis L. subsp. caespitosa (Desf.) Maire

Hém - SA - U3B - Om Op Ms Man MA HA

499. (+) Atractylis serratuloides Sieb.

Ch - A SA - U4B - Op Ms

500. Atractylis cancellata $\mathrm{L}$.

Th - A SA - U3A U3B U4A - Om Op Man Mam MA HA AA

501. Jurinea humilis DC.

Hém - SH H HM - U1A U1B U1C U2A U2E Om MA HA As

502. (-) Carduus chevallieri Bar.

Th - SA - U3B - Op MA HA AA

503. (*) Carduus ballii Hook.

Hém - SH - U2A - MA HA AA

504. Carduus nutans L. $(*)$ subsp. scabrisquamus Jah. Ar.

Hém - SH - U2B - Om R MA HA AA

505. Cirsium casabonae (L.) DC. (*) subsp. dyricolum Maire

Ch - SH H - U2A U2E - MA HA AA

506. Cirsium odontolepis Boiss. Hém - SH H - U2A U2B - R MA

507. (*) Cirsium dyris Jah. \& Maire Hém - SH HM - U1A U1B U1C U2A - MA HA

AA

508. Cirsium flavispina Boiss.

Hém - SH - U2A - R MA HA

509. $(*+)$ Cirsium chrysacanthum (Ball.) Jah. Hém - SA - U3B - HA AA

510. Onopordon acaule $\mathrm{L}$.

Hém - SA SH - U2E U3B - Om R MA HA As

511. (*) Onopordon mesatlanticum Emb. \& Maire Th - SH - U2A - MA

512. $\left({ }^{*}\right)$ Centaurea Benoistii Humb.

Ch - SH - U2E - HA

513. Centaurea tenuifolia Dufour subsp. boissieri

(DC.) Emb. \& Maire

Hém - SA - U3B - R MA

514. Centaurea incana Desf. subsp. ornata (Willd.)

Maire

Hém - SA - U3B - Man MA HA AA

515. Centaurea nana Desf.

Hém - SH H - U1B U2A U2B U2E - Om MA

516. (*) Centaurea litardierei Jah. \& Maire Hém - SH HM - UIB UIC - MA

517. Centaurea theryi Emb. \& Maire Hém - SH HM - UIB - MA

518. (+) Centaurea maroccana Ball.

Th - A SA - U3A U4A - Op Ms Man Mam 
HA AA As

519. Centaurea melitensis $\mathrm{L}$.

Th - A SA - U3A U3B U4A - Om Op Man Mam MA HA AA As

520. Mantisalca salmantica (L.) Briq. \& Cavill.

Hém - SH - U2A - Om Op Man Mam R MA HA AA As

521. Rhaponticum acaule (L.) DC.

Hém - SA - U3B - LM Om Op Man Mam R MA

HA AA

522. Leuzea conifera (L.) DC.

Hém - SA - U3B - Om Man R MA HA

523. (*) Leuzea berardioides Coss.

Hém - HM - UlB UIC - MA HA

524. Carthamus lanatus L. subsp. genuinus (Batt.)

Maire

Th - SA - U3B - Man Mam MA HA AA

525. Carduncellus pinnatus (Desf.) DC. $\left({ }^{*}+\right)$ subsp. lucens Ball.

Hém - HM - U1B - HA

526. Carduncellus atractyloides Batt.

Hém - HM - U1B U1C - MA HA

527. Carduncellus pomelianus Batt. (*) var. mesatlanticus Maire

Hém - SH H HM - U1A U1B U1C U2A U2B

U2E MA

528. Catananche caerulea $\mathrm{L}$.

$\mathrm{G}$ - SH H - U2A U2B U2E - Om Man R

MA HA AA As

529. Catananche caespitosa Desf. Hém - SH HM - U1B - MA HA AA

530. $(+)$ Koelepinia linearis Pallas

Th - A SA - U3B U4A - Op Ms Mam

531. Hedypnois cretica (L.) Willd. subsp. tubiformis

(Ten.) Mur.

Th - SH - U2C - LM Om Op Man Mam R MA

HA AA

532. Leontodon tuberosus $\mathrm{L}$.

Hém - SH HM - U1B U2B - Om Man Mam $\mathrm{R}$ MA HA

533. Leontodon hispanicus Poirret

Hém - SA SH - U1B U2B U3B U2E -

Om Op MA HA

534. $\left({ }^{*}\right)$ Leontodon pitardii Maire

Hém - SA SH - U2E U3B - MA HA

535. Leontodon autumnalis $\mathrm{L}$.

Hém - SH H - U2A U2E - MA HA AA

536. (+) Picris coronopifolia (Desf.) DC.

subsp. albida (Ball.) Maire

Th - A SA - U4B - Ms Man HA AA
537. Tragopogon porrifolius L.

Th - SH - U2A - Man R MA HA

538. Geropogon glaber $\mathrm{L}$.

Th - SH - U2C - Man Mam R MA HA

539. Scorzonera laciniata $\mathrm{L}$.

Th - A SA SH HM - U1B U2C U4A

Om Op Man Mam R MA HA AA

540. Scorzonera undulata Vahl. subsp. deliciosa

(Goss.) Maire

Hém - A SA - U3B U4A - Om Op Man Mam

R MA HA AA

541. Scorzonera pygmaea Sibth. \& Sm.

Hém - SA SH HM - U1A U1B U1C U2B U2E

U3B - Om R MA HA AA

542. Andryala agardhii Haenseler

Ch - HM - U1B UlC - MA

543. Andryala integrifolia $L$.

subsp. eu-integrifolia Maire

Th - SA - U3B - LM Om Op Man Mam R MA

HA AA

544. Taraxacum obovatum (Willd.) DC.

Hém - SH H - U1B U2A - Op Man R MA HA

545. Launaea acanthoclada Maire

Hém - SA - U3B - Op Ms Man R MA HA AA

As

546. Launaea arborescens (Batt.) Maire

Nph - A SA - U4A U4B - Op Ms Man Mam R

MA HA AA As

547. Sonchus tenerrimus L.

subsp. eu-tenerrimus Maire

Th - SA SH - U2A U3B - LM Man Mam R MA

HA AA

548. Sonchus maritimus L.

Hém - SA - U2C - LM Om Op Ms Man Mam R

MA HA AA As

549. Lactuca viminea (L.) Presl.

Hém - SA SH - U2A U3B - Man MA HA AA

550. $(*)$ Lactuca reviersii Litt. \& Maire

Hém - SH HM - UlB - MA

551. Lactuca saligna $\mathrm{L}$.

Th - SH - U2C - Om Op Man Mam R MA HA

552. Lactuca virosa $\mathrm{L}$. (*) subsp. cornigera (Pau. \&

F. Q.) Emb. \& Maire

Hém - SH H - U2A U2B U2C - R MA

553. Lactuca tenerrima Poirret

Hém - SH H - U2A - R MA HA AA

554. Crepis vesicaria L. subsp. taraxacifolia (Thuill.)

Hém - SA - U3A - Om Op Man Mam R MA HA AA 555. (*) Crepis hookeriana Ball. 
Hém - SH HM - UlB - R MA HA

556. Hieracium phlomoides Froel. (*) subsp. mesatlanticum Maire \& Zahn.

Hém. - HM - UlB - MA

557. Hieracium pseudopilosella Ten. (*) subsp. tenuicaule $\mathrm{N}$. $\mathrm{P}$.

Hém - SH H - U1B U2A - MA

\section{BIBLIOGRAPHIE}

EMBERGER, L. -1939- Aperçu général sur la végétation du Maroc, commentaire de la carte phytogéographique du Maroc au 1/1 500000. Veröff. Geobot. Rubel Inst. Zurich 14: 40-157.

EMBERGER, L. \& R. MAIRE -1941-Catalogue des plantes du Maroc. Vol. 4. Minerva, Alger. FENNANE et al. (eds) -1999- Flore pratique du Maroc. Vol. 1. Trav. Inst. Sc., Série botanique, $n^{\circ} 36$, Rabat.

GREUTER, W., H. BURDET \& G. LOG (eds). 1984/1986/1989- Med-Cheklist, inventaire critique des plantes vasculaires des pays circumméditerranéens, vol. 1,2 et 4 , conservatoire et jardin botanique de la ville de Genève.

JAHANDIEZ, E. \& R. MAIRE -1931/1932/1934Catalogue des plantes du Maroc. Vol. 1,2 et 3 . Minerva, Alger.

MAIRE, R. - 1952/1986-Flore de l'Afrique du Nord, 16 Vol., Lechevalier, Paris.

PEYRE, C. -1979-Recherche sur l'étagement de la végétation dans le massif du Bou-Iblane (MoyenAtlas oriental, Maroc) Thèse de $3^{\text {ime }}$ cycle, Univ. Aix Marseille, Fac. Sc. St. Jerôme.

QUEZEL, P. -1957-Peuplement végétal des hautes montagnes de l'Afrique du Nord. Lechevalier, Paris.
QUEZEL, P.\& S. SANTA -1962/63- Nouvelle flore d'Algérie et des régions désertiques méridionales. Vol. 1 et 2.CNRS, Paris.

SAUVAGE, Ch. -1975- L'état actuel de nos connaissances sur la flore du Maroc. In "La flore du bassin méditerranéen: essai de systématique synthétique”, colloque intern. du CNRS, n 235: 131-139.

Aceptado para su publicación en julio de 2002

Adresse de l'auteur. Laboratoire de Biotechnologie végétale. Département de Biologie. Faculté des Sciencies, Meknès. Maroc. 